\title{
Selective Intramolecular Palladium(II)-Catalyzed Aminooxygenation vs. Diamination of Alkenylureas: Efficient Microwave-Assisted Reactions to Bicyclic Piperazinones
}

\author{
Gianluigi Broggini, ${ }^{\mathrm{a}, *}$ Vincenzina Barbera, ${ }^{\mathrm{b}}$ Egle M. Beccalli, ${ }^{\mathrm{c}}$ Ugo Chiacchio, ${ }^{\mathrm{b}}$ \\ Andrea Fasana, ${ }^{\mathrm{a}}$ Simona Galli, ${ }^{\mathrm{a}}$ and Silvia Gazzola ${ }^{\mathrm{a}}$ \\ a Dipartimento di Scienza e Alta Tecnologia, Università dell'Insubria, via Valleggio 11, 22100 Como, Italy \\ Fax: (+39)-(0)31-238-6449; phone: (+39)-(0)31-238-6444; e-mail: gianluigi.broggini@uninsubria.it \\ b Dipartimento di Scienze del Farmaco, Università di Catania, viale Andrea Doria 6, 95125 Catania, Italy \\ c DISFARM, Sezione di Chimica Organica “A. Marchesini”, Università di Milano, via Venezian 21, 20133 Milano, Italy
}

Received: February 2, 2013; Revised: April 18, 2013; Published online: May 15, 2013

Supporting information for this article is available on the WWW under http://dx.doi.org/10.1002/adsc.201300104.

\begin{abstract}
Alkenylureas arising from glycine allylamides were proven to be suitable substrates for the synthesis of bicyclic five-membered ring-fused piperazinones. The reported intramolecular domino processes, performed under oxidative conditions with bis(acetonitrile)palladium dichloride as catalyst and copper(II) chloride in a stoichiometric amount by microwave activation, were completely selective, involving either diamination or aminooxygenation. While the latter process is determined by the direct intervention of the urea oxygen on the $\sigma$-alkylpalladium intermediate, the diamination reaction can in principle derive from a direct attack of the second nitrogen atom on the palladium complex or on the first formed chloromethylpiperazinone. Indeed, this latter species was isolated and proved to be capable of conversion solely into the imidazopiperazinone.
\end{abstract}

Keywords: domino reactions; heterocycles; homogeneous catalysis; microwave chemistry; palladium

\section{Introduction}

The difunctionalization of alkenes under oxidative conditions is a fruitful strategy for the synthesis of various organic compounds. ${ }^{[1]}$ Recent investigations of palladium-catalyzed oxidative reactions have evidenced that domino processes such as aminooxygenations, ${ }^{[2]}$ diaminations, ${ }^{[3]}$ dioxygenations, ${ }^{[4]}$ carboaminations, ${ }^{[5]}$ and aminohalogenations ${ }^{[6]}$ of alkenes can be efficiently used to achieve (poly)functionalized compounds or bicyclic systems.
Alkenylureas have been extensively explored in double intramolecular domino processes providing the formation of oxazole and imidazole bicyclic products either by transition metal-catalyzed reactions, ${ }^{[7,8]}$ or by using iodonium reagents ${ }^{[8]}$ (Figure 1 ). These transformations involve an initial carbon-nitrogen bond formation, followed by the intervention of the oxygen or the second nitrogen atom of the urea group on the intermediate to generate the final structure. Among the cyclizations of alkenylureas promoted by transition metals, Muñiz and co-workers have recently reported a palladium-catalyzed reaction which gives selectively the diamination products working with $\mathrm{PhI}(\mathrm{OAc})_{2}{ }^{[7]}$ or a $\mathrm{Cu}(\mathrm{II})$ salt $^{[8]}$ as oxidants. Conversely, the aminooxygenation process was only occasionally operative when terminally substituted allyl substrates were used. ${ }^{[8]}$ A totally selective aminooxygenation was obtained in the presence of a catalytic system based on platinum chloride. ${ }^{[9]} \mathrm{N}$-Iodosuccinimide-

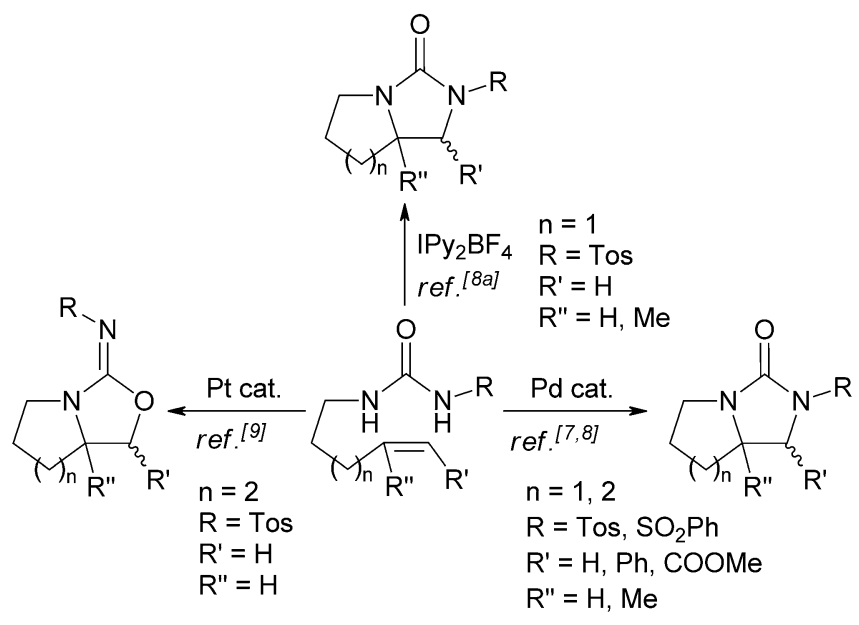

Figure 1. State of the art for the cyclization of alkenylureas. 
<smiles>[R]OC(=O)NCC(=O)N([R])CC=C</smiles>

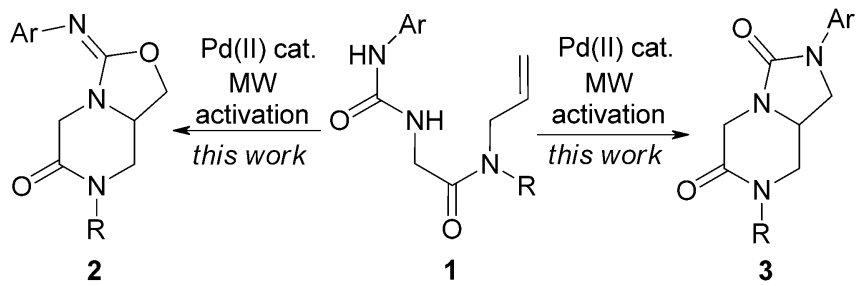

Scheme 1. Palladium-catalyzed double intramolecular difunctionalization of alkenylureas.

mediated reactions were proven to be effective to perform diaminations and aminoalkoxylations of $N$-sulfonyl ureas. ${ }^{[10]}$

As a part of our ongoing interest in constructing hetero-polycyclic compounds by palladium catalysis, ${ }^{[11]}$ we have recently reported the intramolecular aminocarboxylation of tert-butoxycarbonyl-protected aminoalkenes catalyzed by palladium in the presence of copper chloride to produce piperazinone derivatives fused to an oxazolidinone ring (Scheme 1). ${ }^{[2]}$ Herein we describe a selective synthesis of oxazoloand imidazo-piperazinones ( $\mathbf{2}$ and $\mathbf{3}$, respectively) by selective and straightforward $\mathrm{Pd}(\mathrm{II})$-catalyzed microwave-activated aminooxygenation and diamination re- actions of alkenylureas $\mathbf{1}$ arising from glycine allylamides.

\section{Results and Discussion}

Initially, we examined the double intramolecular domino reaction on the $N$-phenylurea 1a, deriving from $N$-allyl- $N$-cyclohexylglycine amide which was chosen as the model. The conditions that successfully promoted the aminocarboxylation process of $\mathrm{N}$-Bocprotected allylic amides of $\alpha$-amino acids, i.e., $\mathrm{PdCl}_{2}$ $(\mathrm{MeCN})_{2} 5 \mathrm{~mol} \%$ and $\mathrm{CuCl}_{2}$ in a stoichiometric amount in DMF at $100{ }^{\circ} \mathrm{C},{ }^{[2 f]}$ resulted in a scarcely selective process, giving a mixture of three products in $39 \%, 32 \%$ and $11 \%$ yields, respectively, following the order of chromatographic elution (Table 1, entry 1). Analytical and spectroscopic data allowed us to identify the aminochlorination product $\mathbf{4 a}$ and the bicyclic ring-fused compounds $2 \mathbf{a}$ and 3a. The distinction between the isomeric oxazolo- and imidazo-piperazine structures 2a and 3a was achieved on the basis of the ${ }^{13} \mathrm{C}$ NMR chemical shifts of the methylene group of the five-membered ring, which resulted to be less shielded for the oxazole ring compared with the imidazole one $(67.9 \mathrm{ppm} v$ s. $44.4 \mathrm{ppm})$, in agreement with the literature data. ${ }^{[12]}$

The outcome of the reaction, although occurring with low selectivity, gave these clues: (i) the $\mathrm{PdCl}_{2}$ $(\mathrm{MeCN})_{2} / \mathrm{CuCl}_{2}$ catalytic system was proven to be suitable for the cyclization of alkenylureas, furnishing piperazinone products by domino processes; (ii) both

Table 1. Optimization of selective reaction conditions.<smiles>C=CCN(C(=O)CNC(=O)Nc1ccccc1)C1CCCCC1</smiles>

1a

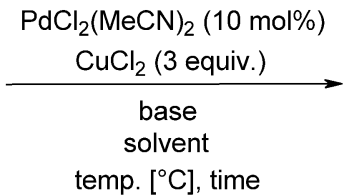

temp. $\left[{ }^{\circ} \mathrm{C}\right]$, time<smiles>O=C1CN(C2CCCCC2)CC2CO/C(=N/c3ccccc3)N12</smiles>

$2 a$<smiles>O=C1CN2C(=O)N(c3ccccc3)CC2CN1C1CCCCC1</smiles>

3a<smiles>O=C1CN(C(=O)Nc2ccccc2)C(CCl)CN1C1CCCCC1</smiles>

$4 a$

\begin{tabular}{|c|c|c|c|c|c|c|c|}
\hline Entry & Base & Solvent & Temperature $\left[{ }^{\circ} \mathrm{C}\right]$ & Time & $\mathbf{2 a}[\%]$ & $\mathbf{3 a}[\%]$ & $\mathbf{4 a}[\%]$ \\
\hline 1 & - & DMF & 100 & $24 \mathrm{~h}$ & 32 & 11 & 39 \\
\hline 2 & - & $\mathrm{THF}$ & 60 & $24 \mathrm{~h}$ & 83 & - & - \\
\hline 3 & $\mathrm{~K}_{2} \mathrm{CO}_{3}$ & DMF & 100 & $24 \mathrm{~h}$ & 65 & 17 & - \\
\hline 4 & $\mathrm{~K}_{2} \mathrm{CO}_{3}$ & $\mathrm{MeCN}$ & reflux & $24 \mathrm{~h}$ & 42 & 9 & - \\
\hline 5 & $\mathrm{AcONa}$ & DMF & 100 & $24 \mathrm{~h}$ & 27 & 13 & - \\
\hline 6 & DBU & DMF & 100 & $24 \mathrm{~h}$ & - & - & - \\
\hline 7 & DIPEA & DMF & 100 & $24 \mathrm{~h}$ & - & - & - \\
\hline 8 & DIPEA & $\mathrm{CH}_{2} \mathrm{Cl}_{2}$ & 40 & $24 \mathrm{~h}$ & - & - & - \\
\hline 9 & $\mathrm{~K}_{2} \mathrm{CO}_{3}$ & DMF & $150^{[\mathrm{a}]}$ & $15 \mathrm{~min}$ & - & 78 & - \\
\hline 10 & $\mathrm{~K}_{2} \mathrm{CO}_{3}$ & DMF & $60^{[\mathrm{a}]}$ & $30 \mathrm{~min}$ & 85 & - & - \\
\hline 11 & - & DMF & $60^{[\mathrm{a}]}$ & $30 \mathrm{~min}$ & 85 & - & - \\
\hline 12 & - & DMF & 60 & $24 \mathrm{~h}$ & 76 & - & - \\
\hline
\end{tabular}

\footnotetext{
[a] MW activation.
} 
oxygen and nitrogen atoms of the urea group can promote the second step of the process giving the divergent formation of a five-membered ring fused to the piperazine moiety; (iii) besides aminooxygenation reactions analogous to those observed with carbamate derivatives, alkenylureas can undergo aminochlorination and diamination processes.

Both $\mathrm{PdCl}_{2}(\mathrm{MeCN})_{2}$ and $\mathrm{CuCl}_{2}$ were proven to be essential for the conversion of 1a to bicyclic products. ${ }^{[2]}$ In order to obtain more selective processes, different parameters including base, solvent and temperature were screened in combination with the established $\mathrm{PdCl}_{2}(\mathrm{MeCN})_{2} / \mathrm{CuCl}_{2}$ catalytic system. The most significant results are collected in Table 1 . The use of THF at $60^{\circ} \mathrm{C}$ gave the aminooxygenation product 2a in $83 \%$ yield (entry 2). Due to the satisfactory behaviour of these conditions to involve the oxygen atom in the domino process, we tested new conditions in the presence of a base to favour the diamination reaction. Although in a few cases the formation of the imidazo[1,5-a]pyrazine product 3a was observed, the use of various bases such as $\mathrm{K}_{2} \mathrm{CO}_{3}$, AcONa, DIPEA and DBU in different solvents did not provide a satisfactory outcome of the diamination process (Table 1, entries 3-8). Conversely, the activation by microwave irradiation highlighted the determinant role of temperature to improve the selectivity in the aminooxygenation and diamination pathways. Firstly, the exposure to the microwaves of a DMF solution of $\mathbf{1 a}$ in the presence of $\mathrm{K}_{2} \mathrm{CO}_{3}$ at $150^{\circ} \mathrm{C}$ for $15 \mathrm{~min}$ led to the diamination product $\mathbf{3 a}$ as the sole product, isolated in $78 \%$ yield (Table 1 , entry 9 ). Working under the same conditions at lower temperature $\left(60 v s .150^{\circ} \mathrm{C}\right)$ for $30 \mathrm{~min}$, only the aminooxygenation compound 2a was observed (Table 1, entry 10). Further investigations revealed that the selectivity in this process is preserved on working in the absence of the base at $60^{\circ} \mathrm{C}$, also by conventional heating (Table 1 , entries 11 and 12).

Comparing the outcomes of the cyclizations reported in Table 1, in particular the conditions of entries 9 and 10 , temperature seems to be determinant for the formation of oxazolyl and imidazolyl compounds. This feature can justify the total selectivity of the diamination product obtained by microwave irradiation working at $150^{\circ} \mathrm{C}$ (entry 10), plausibly due to the rapid heating achieved by this activation technique that avoids the alternative product. On the other hand, the possibility that the oxazolo-piperazine $\mathbf{2 a}$ may convert into 3a by a progressive heating was excluded by the treatment of the former at $150^{\circ} \mathrm{C}$ in DMF, either in the absence or in the presence of the catalytic system.

Based on these results, we verified the scope of the reaction on a wide range of alkenylureas, in all cases by microwave irradiation and preferring the presence of $\mathrm{K}_{2} \mathrm{CO}_{3}$ in the aminooxygenation reactions (entry 10 of Table 1) to shorten the reaction times. Ureas $\mathbf{1 b}-\mathbf{j}$, easily prepared by removal of the tert-butoxycarbonyl group from Boc-glycine allylic amides and subsequent reaction with the appropriate isocyanate, were envisaged as substrates suitable to warrant a satisfactory survey of substituents on the aryl moiety. Aminooxygenation and diamination processes evidenced similar outcomes for all alkenylureas, leading to the bicylic products $\mathbf{2}$ and $\mathbf{3}$ with the yields described in Table 2. It is worth noting that both domino reactions are inhibited by the presence of a substituent such as methyl, phenyl or methoxycarbonyl group on the terminal position of the allylic moiety. The domino processes performed on optically active substrates, such as the $N, N$-allylcyclohexylamide of $\mathrm{L}$-valine and $\mathrm{L}$ phenylalanine, gave in both cases inseparable mixtures of diastereoisomeric bicyclic products.

It should be remarked that the molecular structure of the oxazolo-piperazinone $\mathbf{2 d}$, obtained from the 2-

Table 2. Scope of aminooxygenation and diamination reactions.
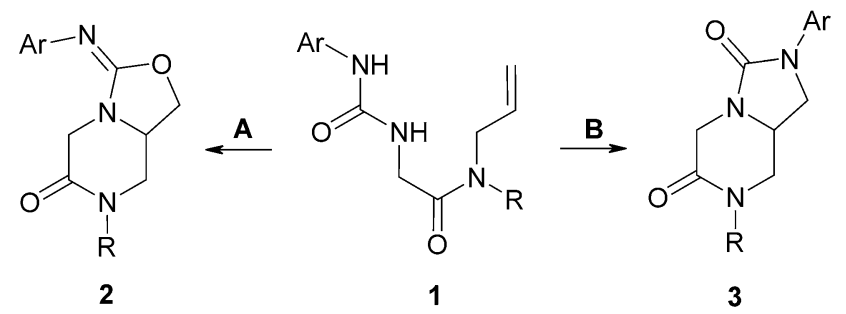

\begin{tabular}{|c|c|c|c|c|c|}
\hline Substrate & $\mathrm{R}$ & $\mathrm{Ar}$ & Conditions $^{[\mathrm{a}]}$ & $2[\%]$ & $3[\%]$ \\
\hline $1 \mathrm{~b}$ & $\mathrm{Cy}$ & $4-\mathrm{Cl}-\mathrm{C}_{6} \mathrm{H}_{4}$ & $\mathbf{A}$ & 75 & \\
\hline $\mathbf{1 b}$ & $\mathrm{Cy}$ & $4-\mathrm{Cl}-\mathrm{C}_{6} \mathrm{H}_{4}$ & $\mathbf{B}$ & & 73 \\
\hline 1c & Cy & $4-\mathrm{NO}_{2}-\mathrm{C}_{6} \mathrm{H}_{4}$ & $\mathbf{A}$ & 71 & \\
\hline 1c & $\mathrm{Cy}$ & $4-\mathrm{NO}_{2}-\mathrm{C}_{6} \mathrm{H}_{4}$ & B & & 78 \\
\hline 1d & $\mathrm{Cy}$ & $2-\mathrm{NO}_{2}-\mathrm{C}_{6} \mathrm{H}_{4}$ & $\mathbf{A}$ & 77 & \\
\hline 1d & $\mathrm{Cy}$ & $2-\mathrm{NO}_{2}-\mathrm{C}_{6} \mathrm{H}_{4}$ & B & & 80 \\
\hline 1e & $\mathrm{Cy}$ & $4-\mathrm{MeO}-\mathrm{C}_{6} \mathrm{H}_{4}$ & $\mathbf{A}$ & 78 & \\
\hline 1e & $\mathrm{Cy}$ & $4-\mathrm{MeO}-\mathrm{C}_{6} \mathrm{H}_{4}$ & B & & 79 \\
\hline 1f & $\mathrm{Bn}$ & $\mathrm{C}_{6} \mathrm{H}_{5}$ & $\mathbf{C}$ & 79 & \\
\hline 1f & $\mathrm{Bn}$ & $\mathrm{C}_{6} \mathrm{H}_{5}$ & $\mathbf{B}$ & & 74 \\
\hline $1 g$ & $\mathrm{Bn}$ & $4-\mathrm{Cl}-\mathrm{C}_{6} \mathrm{H}_{4}$ & $\mathbf{A}$ & 76 & \\
\hline $1 \mathrm{~g}$ & $\mathrm{Bn}$ & 4-Cl- $\mathrm{C}_{6} \mathrm{H}_{4}$ & B & & 72 \\
\hline $1 \mathrm{~h}$ & $\mathrm{Bn}$ & $4-\mathrm{NO}_{2}-\mathrm{C}_{6} \mathrm{H}_{4}$ & $\mathbf{A}$ & 72 & \\
\hline 1h & $\mathrm{Bn}$ & $4-\mathrm{NO}_{2}-\mathrm{C}_{6} \mathrm{H}_{4}$ & $\mathbf{B}$ & & 76 \\
\hline $\mathbf{1 i}$ & $\mathrm{Bn}$ & $2-\mathrm{NO}_{2}-\mathrm{C}_{6} \mathrm{H}_{4}$ & $\mathbf{A}$ & 74 & \\
\hline $\mathbf{1 i}$ & $\mathrm{Bn}$ & $2-\mathrm{NO}_{2}-\mathrm{C}_{6} \mathrm{H}_{4}$ & $\mathbf{B}$ & & 78 \\
\hline $\mathbf{1 j}$ & $\mathrm{Bn}$ & $4-\mathrm{MeO}-\mathrm{C}_{6} \mathrm{H}_{4}$ & $\mathbf{A}$ & 77 & \\
\hline$\dot{\mathbf{1 j}}$ & $\mathrm{Bn}$ & $4-\mathrm{MeO}-\mathrm{C}_{6} \mathrm{H}_{4}$ & $\mathbf{B}$ & & 73 \\
\hline
\end{tabular}
( $3 \mathrm{mmol})$ and urea $(1 \mathrm{mmol}), \mathrm{DMF}, 60^{\circ} \mathrm{C}$, MW irradiation, $30 \mathrm{~min}$; conditions $\mathbf{B}$ : $\mathrm{PdCl}_{2}\left(\mathrm{MeCN}_{2}(10 \mathrm{~mol} \%)\right.$, $\mathrm{CuCl}_{2}(3 \mathrm{mmol}), \mathrm{K}_{2} \mathrm{CO}_{3}(3 \mathrm{mmol})$, and urea $(1 \mathrm{mmol})$, $\mathrm{DMF}, 150^{\circ} \mathrm{C}$, MW irradiation, $15 \mathrm{~min}$; conditions $\mathbf{C}$ : $\mathrm{PdCl}_{2}(\mathrm{MeCN})_{2} \quad(10 \mathrm{~mol} \%), \mathrm{CuCl}_{2}(3 \mathrm{mmol})$ and urea (1 mmol), THF, $60^{\circ} \mathrm{C}, 24 \mathrm{~h}$. 


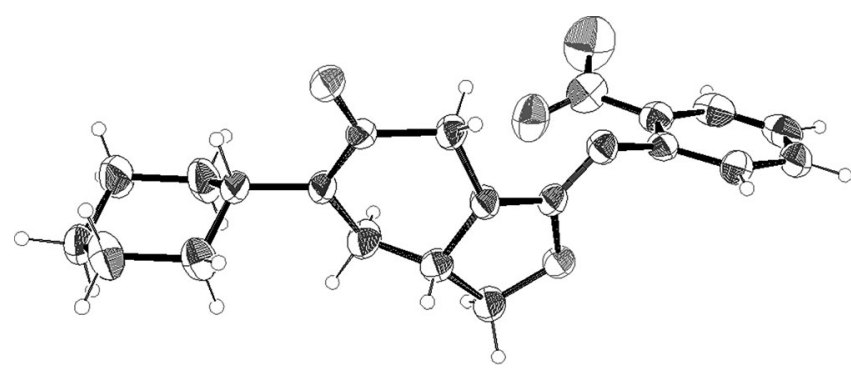

Figure 2. Ortep drawing, at $30 \%$ probability level, of the molecular structure of species $\mathbf{2 d}$.

nitrophenyl-substituted urea 1d by performing the reaction at $60^{\circ} \mathrm{C}$ under microwave activation, was confirmed by a single crystal X-ray diffraction study (Figure 2). ${ }^{[13]}$

Having in hand the aminochlorination product $4 \mathbf{a}$, we planned some experiments to find indications on its possible role as the precursor for the oxazole and imidazole bicyclic products 2 and 3. ${ }^{[14]}$ Firstly, chloromethylpiperazinone 4a was treated under the conditions which were successful in the aminooxygenation process [i.e., $\mathrm{PdCl}_{2}(\mathrm{MeCN})_{2} / \mathrm{CuCl}_{2}$ as the catalytic system in $\mathrm{THF}$ at $60^{\circ} \mathrm{C}$ with conventional heating, or in $\mathrm{DMF}$ at $60^{\circ} \mathrm{C}$ for $30 \mathrm{~min}$ under microwave activation], but no reaction was observed (Scheme 2). The

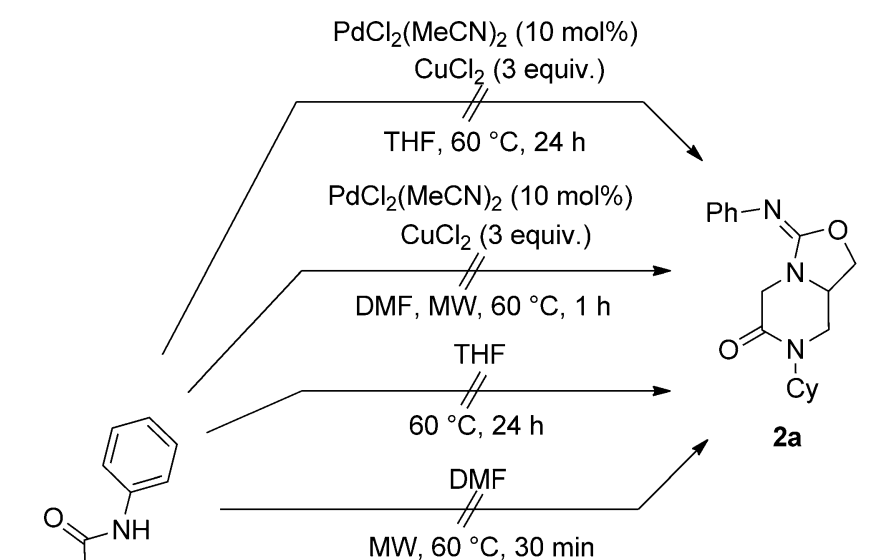

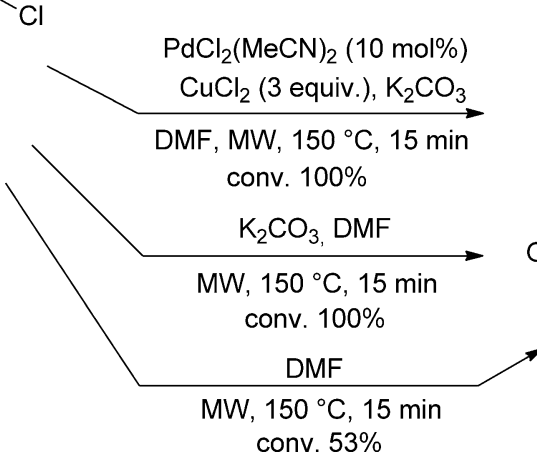

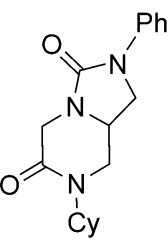

$3 a$ conv. $53 \%$

Scheme 2. Attempts to promote oxygenation and amination reactions from the chloromethyl derivative $\mathbf{4 a}$.

conversion of compound $\mathbf{4 a}$ was unsuccessful also on heating in THF or by treatment under microwave irradiation in DMF in the absence of the couple $\mathrm{PdCl}_{2}(\mathrm{MeCN})_{2} / \mathrm{CuCl}_{2}$. Conversely, on performing the reaction with the catalytic system in $\mathrm{DMF}$ at $150^{\circ} \mathrm{C}$ under microwave activation, the chloro derivative $4 \mathbf{a}$ was quantitatively converted into the imidazo-piperazinone product 3a. The transformation into the diamination product was achievable also without $\mathrm{Pd}$ catalyst and $\mathrm{CuCl}_{2}$, even if the presence of $\mathrm{K}_{2} \mathrm{CO}_{3}$ was required to have a quantitative yield.

This behaviour was confirmed by submitting different chloromethylpiperazinones to aminooxygenation and diamination reactions, as described in Table 3. Compounds $\mathbf{4 f}, \mathbf{h}, \mathbf{j}$ were converted solely into the imidazo-fused products $\mathbf{3 f}, \mathbf{h}, \mathbf{j}$, isolated in all cases in high yields.

This investigation on the reactivity of the chloromethylpiperazinones furnished new insights on the mechanism of the double intramolecular domino processes. Even if the conversion of the compound $\mathbf{1}$ into the imidazole bicyclic product $\mathbf{3}$ can involve the chloro intermediate 4 or a direct intervention of the nitrogen atom on the $\sigma$-alkyl-complex $\mathbf{A}$, the aminooxygenation process can only arise from an intramolecular nucleophilic attack by the oxygen atom of the urea on the $\sigma$-alkyl-complex A' (Figure 3). In both pathways, $\mathrm{CuCl}_{2}$ would inhibit the common palladium $\beta$-hydride elimination favouring the loss of the $\mathrm{Pd}$ species through a transient palladium oxidation ${ }^{[15]}$ or by formation of a heterobimetallic $\sigma-\mathrm{Pd} / \mathrm{Cu}$ complex. ${ }^{[16]}$ The

Table 3. Control experiments proving the role of chloromethyl derivatives as possible intermediates toward derivatives 3 .

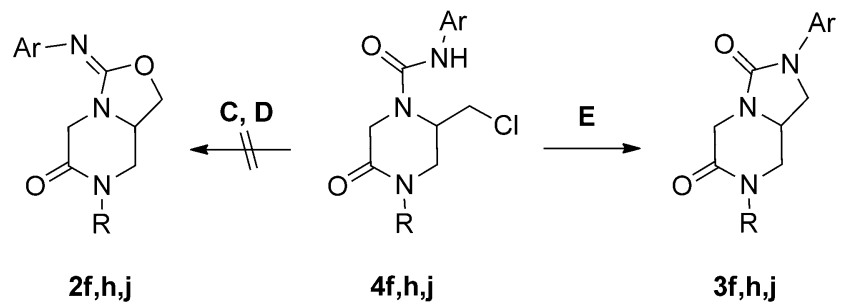

\begin{tabular}{|c|c|c|c|c|c|}
\hline Substrate & $\mathrm{R}$ & Ar & Conditions $^{[\mathrm{a}]}$ & $2[\%]$ & $3[\%]$ \\
\hline $4 f$ & $\mathrm{Bn}$ & $\mathrm{C}_{6} \mathrm{H}_{5}$ & $\mathbf{E}$ & - & 92 \\
\hline 4f & $\mathrm{Bn}$ & $\mathrm{C}_{6} \mathrm{H}_{5}$ & $\mathbf{C}, \mathbf{D}$ & - & - \\
\hline 4h & $\mathrm{Bn}$ & $4-\mathrm{NO}_{2}-\mathrm{C}_{6} \mathrm{H}_{4}$ & $\mathbf{E}$ & - & 97 \\
\hline $4 h$ & $\mathrm{Bn}$ & $4-\mathrm{NO}_{2}-\mathrm{C}_{6} \mathrm{H}_{4}$ & $\mathbf{C}, \mathbf{D}$ & - & - \\
\hline $4 \mathbf{j}$ & $\mathrm{Bn}$ & $4-\mathrm{MeO}-\mathrm{C}_{6} \mathrm{H}_{4}$ & $\mathbf{E}$ & - & 89 \\
\hline $\mathbf{4 j}$ & $\mathrm{Bn}$ & $4-\mathrm{MeO}-\mathrm{C}_{6} \mathrm{H}_{4}$ & $\mathbf{C}, \mathbf{D}$ & - & - \\
\hline
\end{tabular}

( $3 \mathrm{mmol})$ and urea $(1 \mathrm{mmol}), \mathrm{THF}, 60^{\circ} \mathrm{C}, 24 \mathrm{~h}$; conditions D: $\mathrm{PdCl}_{2}(\mathrm{MeCN})_{2}(10 \mathrm{~mol} \%), \mathrm{CuCl}_{2}(3 \mathrm{mmol})$, and urea $(1 \mathrm{mmol}), \mathrm{DMF}, 60^{\circ} \mathrm{C}$, MW irradiation, $1 \mathrm{~h}$; conditions E: $\mathrm{K}_{2} \mathrm{CO}_{3}(3 \mathrm{mmol})$ and urea $(1 \mathrm{mmol}), \mathrm{DMF}, 150^{\circ} \mathrm{C}$, MW irradiation, 15 min. 


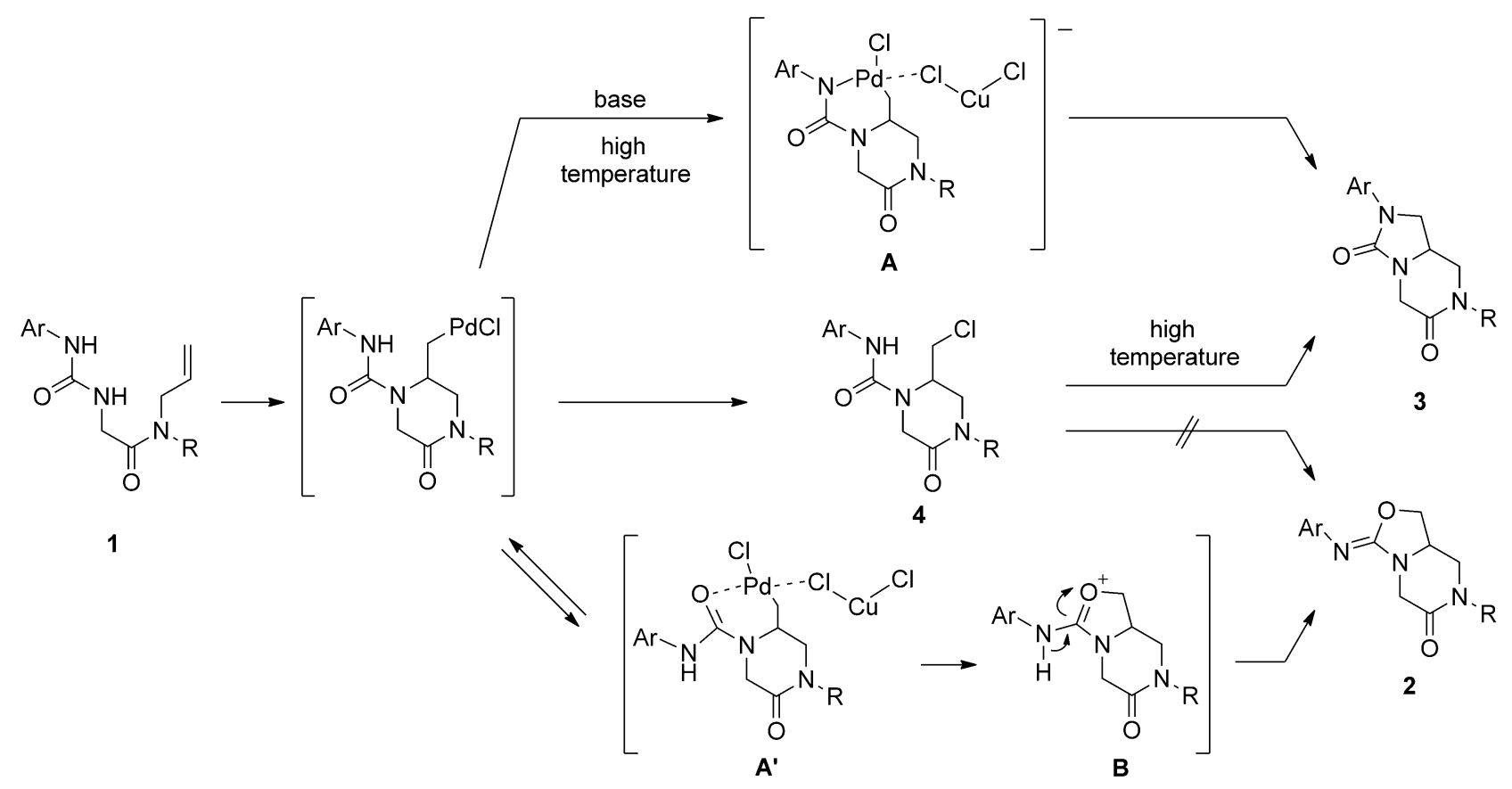

Figure 3. Proposed mechanism for the $\mathrm{Pd}(\mathrm{II}) / \mathrm{CuCl}_{2}$-mediated aminooxygenation and diamination reactions.

aminooxygenation process generates the intermediate B and the elimination of $\mathrm{H}^{+}$furnishes the product 2 . The domino processes plausibly occur by a selective trans-amino-palladation, as proven by studies conducted on deuterated unfunctionalized aminoalkenes. ${ }^{[7 a]}$

In the attempt to convert the chloro derivatives into the bicyclic products, the results observed matched the hypothesis proposed about the cyclization of the analogous alkenyl carbamates, ${ }^{[2]]}$ where chloromethyl-substituted products were never detected, making plausible a direct attack of the oxygen atom of the tert-butoxycarbonyl group on the palladium intermediate.

\section{Conclusions}

In conclusion, we have developed selective double intramolecular aminooxygenation and diamination reactions of alkenylureas to yield bicyclic products containing a piperazinone ring. These $\mathrm{Pd}(\mathrm{II})$-catalyzed transformations under microwave irradiation provide efficient and selective synthetic protocols for the construction of 3-arylimino-oxazolo[3,4-a]pyrazin-6-one and 2-aryl-imidazo[1,5- $a$ ]pyrazin-3,6-dione derivatives in few steps from readily available precursors. The aminooxygenation process can also be carried out in satisfactory yield by conventional heating, even if the reaction times are longer. From the mechanistic point of view, the reactions performed on the chloromethylpiperazinones allowed us to exclude their involve- ment as intermediates in the aminooxygenation pathway.

\section{Experimental Section}

\section{General Remarks}

Reagents and solvents were used as received from commercial sources. Flash column chromatography was performed employing 230-400 mesh silica gel. Analytical thin layer chromatography was performed on silica gel 60 F254. Melting points were measured with a Büchi B-540 apparatus and are uncorrected. IR spectra were recorded on a 550 Nicolet FT-IR spectrophotometer. Nuclear magnetic resonance spectra were acquired on an AVANCE 400 Bruker spectrometer operating at $400 \mathrm{MHz}$ for ${ }^{1} \mathrm{H}$ NMR and $100 \mathrm{MHz}$ for ${ }^{13} \mathrm{C}$ NMR. ${ }^{13} \mathrm{C}$ spectra were ${ }^{1} \mathrm{H}$ decoupled and multiplicities were determined by the APT pulse sequence. Mass spectra were determined on an HPLC-MS LCQ-Advantage Thermo Finnigan instrument. Elemental analyses were executed on a PerkinElmer CHN Analyzer Series II 2400.

Detailed procedures for the preparation of alkenylureas and details on the X-ray diffraction measurement and analysis are available as Supporting Information.

\section{General Procedure for the Aminooxygenation Reactions}

A mixture of $\mathrm{PdCl}_{2}(\mathrm{MeCN})_{2}(0.1 \mathrm{mmol}), \mathrm{CuCl}_{2}(3 \mathrm{mmol})$ and urea $1(1 \mathrm{mmol})$ in DMF $(10 \mathrm{~mL})$ was heated at $60^{\circ} \mathrm{C}$ for $30 \mathrm{~min}$ under microwave irradiation. Brine $(15 \mathrm{~mL})$ was added and the mixture was extracted with $\mathrm{CH}_{2} \mathrm{Cl}_{2}$ (3× $15 \mathrm{~mL}$ ). The organic phase was dried over $\mathrm{Na}_{2} \mathrm{SO}_{4}$ and the solvent removed under reduced pressure. The crude mixture 
was purified by silica gel column chromatography (petroleum ether/AcOEt 3:7).

7-Cyclohexyl-3-(phenylimino)-1,7,8,8a-tetrahydro-3Hoxazolo[3,4-a]pyrazine-6(5H)-one (2a): Yield: $85 \%$; white solid; $\mathrm{mp} 156{ }^{\circ} \mathrm{C}$. IR: $\quad v=1757, \quad 1585 \mathrm{~cm}^{-1} ; \quad{ }^{1} \mathrm{H}$ NMR $\left(400 \mathrm{MHz}, \mathrm{CDCl}_{3}\right): \delta=1.25-1.82\left[\mathrm{~m}, 10 \mathrm{H},\left(\mathrm{CH}_{2}\right)_{5}\right], 3.15(\mathrm{dd}$ $1 \mathrm{H}, J=11.1,9.8 \mathrm{~Hz}, \mathrm{NCHH}), 3.28(\mathrm{dd}, 1 \mathrm{H}, J=11.1,3.8 \mathrm{~Hz}$, $\mathrm{NCH} H), 3.82-3.87(\mathrm{~m}, 1 \mathrm{H}, \mathrm{NCH}), 3.88(\mathrm{~d}, J=17.8 \mathrm{~Hz}, 1 \mathrm{H}$, $\mathrm{CHH}), 4.02(\mathrm{dd}, 1 \mathrm{H}, J=8.6,4.4 \mathrm{~Hz}, \mathrm{OCH} H), 4.43(\mathrm{dd}, 1 \mathrm{H}$, $J=8.6,7.4 \mathrm{~Hz}, \mathrm{OCHH}), 4.46-4.52\left[\mathrm{~m}, 1 \mathrm{H}, \mathrm{NCH}\left(\mathrm{CH}_{2}\right)_{5}\right]$, $4.53(\mathrm{~d}, 1 \mathrm{H}, J=17.8 \mathrm{~Hz}, \mathrm{CHH}) 6.89-6.98(\mathrm{~m}, 1 \mathrm{H}, \operatorname{Ar} H)$, $7.05(\mathrm{~d}, 2 \mathrm{H}, J=7.6 \mathrm{~Hz}, \operatorname{Ar} H), 7.20-7.24(\mathrm{~m}, 2 \mathrm{H}, \operatorname{Ar} H)$; ${ }^{13} \mathrm{C} \mathrm{NMR}\left(100 \mathrm{MHz}, \mathrm{CDCl}_{3}\right): \delta=25.3(\mathrm{t}), 25.4(\mathrm{t}), 25.6(\mathrm{t})$, $29.3(\mathrm{t}), 29.6(\mathrm{t}), 43.6(\mathrm{t}), 46.4(\mathrm{t}), 51.6(\mathrm{~d}), 52.6(\mathrm{~d}), 67.9(\mathrm{t})$ 122.5 (d), 124.4 (d), 128.5 (d), 146.6 (s), 150.9 (s), 164.3 (s); MS: $m / z=313\left(\mathrm{M}^{+}\right)$; anal. calcd. for $\mathrm{C}_{18} \mathrm{H}_{23} \mathrm{~N}_{3} \mathrm{O}_{2}: \mathrm{C} 68.98, \mathrm{H}$ 7.40, N 13.41; found: C 69.17, H 7.14, N 13.50.

7-Cyclohexyl-3-(4-chlorophenylimino),1,7,8,8a-tetrahydro3H-oxazolo[3,4-a]pyrazine-6(5H)-one (2b): Yield: $75 \%$ white solid; $\mathrm{mp} 103^{\circ} \mathrm{C}$. IR: $v=1755,1588,1089 \mathrm{~cm}^{-1}$; ${ }^{1} \mathrm{H} \mathrm{NMR} \quad\left(400 \mathrm{MHz}, \quad \mathrm{CDCl}_{3}\right): \delta=1.33-1.84 \quad[\mathrm{~m}, \quad 10 \mathrm{H}$ $\left.\left(\mathrm{CH}_{2}\right)_{5}\right], 3.23(\mathrm{dd}, 1 \mathrm{H}, J=11.1,9.2 \mathrm{~Hz}, \mathrm{NCHH}), 3.35$ (dd, $1 \mathrm{H}, J=11.1,3.8 \mathrm{~Hz}, \mathrm{NCH} H), 3.95(\mathrm{~d}, 1 \mathrm{H}, J=18.2 \mathrm{~Hz}$, $\mathrm{CHH}), 3.95-4.02(\mathrm{~m}, 1 \mathrm{H}, \mathrm{NCH}), 4.11(\mathrm{dd}, 1 \mathrm{H}, J=8.4$, $4.3 \mathrm{~Hz}, \mathrm{OCH}), 4.51-5.56\left(\mathrm{~m}, 3 \mathrm{H}, \mathrm{OCH} H, \mathrm{NCH}\left(\mathrm{CH}_{2}\right)_{5}\right.$, $\mathrm{CHH}), 7.01(\mathrm{~d}, 2 \mathrm{H}, J=8.6 \mathrm{~Hz}, \operatorname{Ar} H), 7.19(\mathrm{~d}, 2 \mathrm{H}, J=$ $8.7 \mathrm{~Hz}, \mathrm{Ar} H) ;{ }^{13} \mathrm{C}$ NMR $\left(100 \mathrm{MHz}, \mathrm{CDCl}_{3}\right): \delta=25.3(\mathrm{t}), 25.4$ $(\mathrm{t}), 25.5(\mathrm{t}), 29.3(\mathrm{t}), 29.7(\mathrm{t}), 43.7(\mathrm{t}), 46.3(\mathrm{t}), 51.7(\mathrm{~d}), 52.6$ (d), $68.0(\mathrm{t}), 124.7(\mathrm{~d}), 128.7$ (d), $145.0(\mathrm{~s}), 147.2$ (s), 151.1 (s), 164.2 (s); MS: $m / z=347 \quad\left(\mathrm{M}^{+}\right)$; anal. calcd. for $\mathrm{C}_{18} \mathrm{H}_{22} \mathrm{ClN}_{3} \mathrm{O}_{2}$ : C 62.15, H 6.37, N 12.08; found: C 61.91, $\mathrm{H}$ 6.61, N 12.31 .

7-Cyclohexyl-3-(4-nitrophenylimino)-1,7,8,8a-tetrahydro$3 \boldsymbol{H}$-oxazolo[3,4-a]pyrazine-6(5H)-one (2c): Yield: $71 \%$; yellow solid; $\mathrm{mp} 118^{\circ} \mathrm{C}$. IR: $v=1750,1581,1522,1349 \mathrm{~cm}^{-1}$, ${ }^{1} \mathrm{H} \mathrm{NMR} \quad\left(400 \mathrm{MHz}, \quad \mathrm{CDCl}_{3}\right): \delta=1.17-1.83 \quad[\mathrm{~m}, \quad 10 \mathrm{H}$ $\left.\left(\mathrm{CH}_{2}\right)_{5}\right], 3.26(\mathrm{dd}, 1 \mathrm{H}, J=11.0,9.2 \mathrm{~Hz}, \mathrm{NCHH}), 3.43(\mathrm{dd}$, $1 \mathrm{H}, J=11.0,3.8 \mathrm{~Hz}, \mathrm{NCHH}), 3.97(\mathrm{~d}, 1 \mathrm{H}, J=18.3 \mathrm{~Hz}$ $\mathrm{CHH}), 3.98-4.11(\mathrm{~m}, 1 \mathrm{H}, \mathrm{NCH}), 4.20(\mathrm{dd}, 1 \mathrm{H}, J=8.6$, $4.5 \mathrm{~Hz}, \mathrm{OCHH}), 4.57$ (d, $1 \mathrm{H}, J=18.3 \mathrm{~Hz}, \mathrm{CH} H), 4.61-4.65$ $\left(\mathrm{m}, 2 \mathrm{H}, \mathrm{OCH} H, \mathrm{NCH}\left(\mathrm{CH}_{2}\right)_{5}\right), 7.16(\mathrm{~d}, 2 \mathrm{H}, J=9.0 \mathrm{~Hz}$, $\operatorname{Ar} H), 8.11(\mathrm{~d}, 2 \mathrm{H}, J=8.9 \mathrm{~Hz}, \operatorname{Ar} H) ;{ }^{13} \mathrm{C}$ NMR $(100 \mathrm{MHz}$, $\left.\mathrm{CDCl}_{3}\right): \delta=25.3(\mathrm{t}), 25.4(\mathrm{t}), 25.5(\mathrm{t}), 29.3(\mathrm{t}), 29.7(\mathrm{t}), 43.8$ $(\mathrm{t}), 46.1(\mathrm{t}), 51.7(\mathrm{~d}), 52.6(\mathrm{~d}), 68.4(\mathrm{t}), 123.8(\mathrm{~d}), 125.0(\mathrm{~d})$, 142.6 (s), 151.9 (s), 153.4 (s), 164.2 (s); MS: $m / z=358\left(\mathrm{M}^{+}\right)$ anal. calcd. for $\mathrm{C}_{18} \mathrm{H}_{22} \mathrm{~N}_{4} \mathrm{O}_{4}$ : C 60.32, H 6.19, N 15.63; found: C 60.39, H 6.32, N 15.49

7-Cyclohexyl-3-(2-nitrophenylimino)-1,7,8,8a-tetrahydro3H-oxazolo[3,4-a $]$ pyrazine-6(5H)-one (2d): Yield: 77\%; yellow solid; mp $126^{\circ} \mathrm{C}$. IR: $v=1754,1579,1492,1333 \mathrm{~cm}^{-1}$; ${ }^{1} \mathrm{H} N M R \quad\left(400 \mathrm{MHz}, \quad \mathrm{CDCl}_{3}\right): \delta=1.18-1.84 \quad[\mathrm{~m}, \quad 10 \mathrm{H}$ $\left.\left(\mathrm{CH}_{2}\right)_{5}\right], 3.35-3.38(\mathrm{~m}, 2 \mathrm{H}, \mathrm{NCH}, \mathrm{NCH} H), 3.98(\mathrm{~d}, 1 \mathrm{H}$, $J=18.2 \mathrm{~Hz}, \mathrm{CHH}), 4.01-4.05(\mathrm{~m}, 1 \mathrm{H}, \mathrm{NCH}), 4.08(\mathrm{dd}, 1 \mathrm{H}$, $J=8.6, \quad 3.8 \mathrm{~Hz}, \mathrm{OCH} H), 4.46(\mathrm{dd}, 1 \mathrm{H}, J=8.6,7.2 \mathrm{~Hz}$, $\mathrm{OCHH}), 4.53-4.55\left(\mathrm{~m}, 1 \mathrm{H}, \mathrm{NCH}\left(\mathrm{CH}_{2}\right)_{5}\right), 4,56(\mathrm{~d}, 1 \mathrm{H}, J=$ $18.2 \mathrm{~Hz}, \mathrm{CH} H)$ 7.04-7.12 (m, 2H, ArH), 7.42-7.46 (m, $1 \mathrm{H}$ $\operatorname{Ar} H), \quad 7.87(\mathrm{dd}, \quad 1 \mathrm{H}, \quad J=8.4, \quad 1.4 \mathrm{~Hz}, \quad \operatorname{Ar} H) ;{ }^{13} \mathrm{C}$ NMR $\left(100 \mathrm{MHz}, \mathrm{CDCl}_{3}\right): \delta=25.3(\mathrm{t}), 25.4(\mathrm{t}), 25.5(\mathrm{t}), 29.3(\mathrm{t})$, $29.7(\mathrm{t}), 43.9(\mathrm{t}), 46.3(\mathrm{t}), 52.4(\mathrm{~d}), 52.6(\mathrm{~d}), 67.8(\mathrm{t}), 122.4$ (d),124.9 (d), 126.6 (d),133.3 (d), 142.4 (s), 142.8 (s), 152.5 (s), 163.8 (s); MS: $m / z=358\left(\mathrm{M}^{+}\right)$; anal. calcd. for
$\mathrm{C}_{18} \mathrm{H}_{22} \mathrm{~N}_{4} \mathrm{O}_{4}$ : C 60.32, H 6.19, N 15.63; found: $\mathrm{C} 60.09, \mathrm{H}$ 6.41, N 15.70.

7-Cyclohexyl-3-(4-methoxyphenylimino)-1,7,8,8a-tetrahydro-3H-oxazolo[3,4-a $]$ pyrazine-6(5H)-one $(2 \mathrm{e})$ : Yield: $78 \%$; white solid; $\mathrm{mp} 129^{\circ} \mathrm{C}$. IR: $v=1749,1589,1260 \mathrm{~cm}^{-1}$; ${ }^{1} \mathrm{H} \mathrm{NMR} \quad\left(400 \mathrm{MHz}, \quad \mathrm{CDCl}_{3}\right): \delta=1.25-1.83 \quad[\mathrm{~m}, \quad 10 \mathrm{H}$, $\left.\left(\mathrm{CH}_{2}\right)_{5}\right], 3.22(\mathrm{dd}, 1 \mathrm{H}, J=11.1,9.3 \mathrm{~Hz}, \mathrm{NCHH}), 3.34(\mathrm{dd}$, $1 \mathrm{H}, J=11.1,3.8 \mathrm{~Hz}, \mathrm{NCH} H), 3.76\left(\mathrm{~s}, 3 \mathrm{H}, \mathrm{OCH}_{3}\right), 3.89-3.93$ $(\mathrm{m}, 1 \mathrm{H}, \mathrm{NCH}), 3.91(\mathrm{~d}, 1 \mathrm{H}, J=18.2 \mathrm{~Hz}, \mathrm{CHH}), 4.06(\mathrm{dd}$, $1 \mathrm{H}, J=8.6,4.5 \mathrm{~Hz}, \mathrm{OCHH}), 4.47(\mathrm{dd}, 1 \mathrm{H}, J=8.6,7.3 \mathrm{~Hz}$, $\mathrm{OCH} H), 4.49-4.51\left(\mathrm{~m}, 1 \mathrm{H}, \mathrm{NCH}\left(\mathrm{CH}_{2}\right)_{5}\right), 4.54(\mathrm{~d}, 1 \mathrm{H}, J=$ $18.2 \mathrm{~Hz}, \mathrm{CHH}), 6.80(\mathrm{~d}, 2 \mathrm{H}, J=8.9 \mathrm{~Hz}, \operatorname{ArH}), 7.02(\mathrm{~d}, 2 \mathrm{H}$, $J=8.9 \mathrm{~Hz}, \operatorname{ArH}) ;{ }^{13} \mathrm{C}$ NMR $\left(100 \mathrm{MHz}, \mathrm{CDCl}_{3}\right): \delta=25.3(\mathrm{t})$, $25.5(\mathrm{t}), 25.5(\mathrm{t}), 29.3(\mathrm{t}), 29.7(\mathrm{t}), 43.6(\mathrm{t}), 46.5(\mathrm{t}), 51.7(\mathrm{~d})$, $52.5(\mathrm{~d}), 55.4(\mathrm{q}), 67.8(\mathrm{t}), 113.9$ (d), $124.1(\mathrm{~d}), 139.6(\mathrm{~s})$, 150.7 (s), 155.2 (s), 164.4 (s); MS: $m / z=343\left(\mathrm{M}^{+}\right)$; anal.

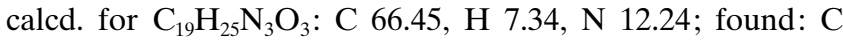
66.38, H 7.62, N 12.47 .

7-Benzyl-3-(phenylimino)-1,7,8,8a-tetrahydro-3H-oxazolo[3,4-a]pyrazine-6(5H)-one (2f): Yield: $79 \%$; colourless oil. IR: $v=1758,1587 \mathrm{~cm}^{-1} ;{ }^{1} \mathrm{H}$ NMR $\left(400 \mathrm{MHz}, \mathrm{CDCl}_{3}\right): \delta=$ $3.26(\mathrm{dd}, 1 \mathrm{H}, J=10.9,4.0 \mathrm{~Hz}, \mathrm{NCHH}), 3.36(\mathrm{dd}, 1 \mathrm{H}, J=$ $10.9,9.4 \mathrm{~Hz}, \mathrm{NCH} H), 3.95-4.03(\mathrm{~m}, 3 \mathrm{H}, \mathrm{OCH}, \mathrm{CH}$, $\mathrm{NCH}), 4.41-4.46(\mathrm{~m}, 1 \mathrm{H}, \mathrm{OCH} H), 4.46(\mathrm{~d}, 1 \mathrm{H}, J=14.2 \mathrm{~Hz}$, $\mathrm{CHHPh}), 4.67(\mathrm{~d}, 1 \mathrm{H}, J=18.3 \mathrm{~Hz}, \mathrm{CH} H), 4.88(\mathrm{~d}, 1 \mathrm{H}, J=$ 14.2 Hz, CHHPh), 7.00-7.07 (m, 2H, ArH) 7.50-7.23 (m, $8 \mathrm{H}, \operatorname{Ar} H) ;{ }^{13} \mathrm{C}$ NMR $\left(100 \mathrm{MHz}, \mathrm{CDCl}_{3}\right): \delta=46.1(\mathrm{t}), 48.4$ $(\mathrm{t}), 50.4(\mathrm{t}), 51.3(\mathrm{~d}), 67.7(\mathrm{t}), 122.7(\mathrm{~d}), 124.0(\mathrm{~d}), 128.0(\mathrm{~d})$, 128.2 (d),128.6 (d), 128.9 (d), 139.7 (s), 146.4(s), 150.7 (s), 165.1 (s); MS: $m / z=321\left(\mathrm{M}^{+}\right)$; anal. calcd. for $\mathrm{C}_{19} \mathrm{H}_{19} \mathrm{~N}_{3} \mathrm{O}_{2}$ : C 71.01, H 5.96, N 13.08; found: C 70.83, H 6.25, N 13.21.

7-Benzyl-3-(4-chlorophenylimino)-1,7,8,8a-tetrahydro-3Hoxazolo[3,4-a]pyrazine-6(5H)-one (2g): Yield: 76\%; white solid; mp $107^{\circ} \mathrm{C}$. IR: $v=1748,1584,1098 \mathrm{~cm}^{-1}$; ${ }^{1} \mathrm{H}$ NMR $\left(400 \mathrm{MHz}, \mathrm{CDCl}_{3}\right): \delta=3.26(\mathrm{dd}, 1 \mathrm{H}, \quad J=11.3, \quad 3.8 \mathrm{~Hz}$, $\mathrm{NCHH}), 3.48(\mathrm{dd}, 1 \mathrm{H}, J=11.3,9.1 \mathrm{~Hz}, \mathrm{NCH} H), 4.00(\mathrm{~d}$, $1 \mathrm{H}, J=18.6 \mathrm{~Hz}, \mathrm{CHH}), 4.01-4.02(\mathrm{~m}, 2 \mathrm{H}, \mathrm{OCH}, \mathrm{NCH})$, 4.44-4.48 (m, 1H, OCH $), 4.47(\mathrm{~d}, 1 \mathrm{H}, J=14.4 \mathrm{~Hz}$, CHHPh), 4.64 (d, $1 \mathrm{H}, J=18.2 \mathrm{~Hz}, \mathrm{CH} H), 4.88$ (d, $1 \mathrm{H}, J=$ $14.4 \mathrm{~Hz}, \mathrm{CH} H \mathrm{Ph}$ ), 7.00 (d, 2H, $J=8.7 \mathrm{~Hz}, \operatorname{Ar} H$ ), 7.19 (d, $2 \mathrm{H}, J=8.6 \mathrm{~Hz}, \operatorname{Ar} H)$ 7.27-7.32 (m, 6H, ArH); ${ }^{13} \mathrm{C}$ NMR $\left(100 \mathrm{MHz}, \mathrm{CDCl}_{3}\right): \delta=44.5(\mathrm{t}), 48.4(\mathrm{t}), 50.4(\mathrm{t}), 51.2(\mathrm{~d})$, $67.8 \quad(\mathrm{t}), 118.5 \quad(\mathrm{~s}), 124.7 \quad(\mathrm{~d}), 127.7 \quad(\mathrm{~d}), 128.0 \quad(\mathrm{~d}), 128.3$ (d),128.5 (d), 128.9 (d), 135.8 (s), 144.9 (s), 150.9 (s), 164.9 (s); MS: $m / z=335\left(\mathrm{M}^{+}\right)$; anal. calcd. for $\mathrm{C}_{19} \mathrm{H}_{18} \mathrm{ClN}_{3} \mathrm{O}_{2}$ : $\mathrm{C}$ 64.13, H 5.10, N 11.81; found: C 64.20, H 4.85, N 11.51.

7-Benzyl-3-(4-nitrophenylimino)-1,7,8,8a-tetrahydro-3Hoxazolo[3,4-a]pyrazine-6(5H)-one (2h): Yield: $72 \%$; pale yellow solid; $\mathrm{mp} 124^{\circ} \mathrm{C}$. IR: $v=1760,1588,1519,1340 \mathrm{~cm}^{-1}$; ${ }^{1} \mathrm{H}$ NMR $\left(400 \mathrm{MHz}, \mathrm{CDCl}_{3}\right): \delta=3.32(\mathrm{dd}, 1 \mathrm{H}, \quad J=11.2$, $4.0 \mathrm{~Hz}, \mathrm{NCHH}), 3.39$ (dd, $1 \mathrm{H}, J=11.2,9.2 \mathrm{~Hz}, \mathrm{NCH} H), 4.00$ $(\mathrm{d}, 1 \mathrm{H}, J=18.4 \mathrm{~Hz}, \mathrm{CHH}), 4.06-4.13(\mathrm{~m}, 2 \mathrm{H}, \mathrm{OCH} H$, $\mathrm{NCH}), 4.47$ (d, $1 \mathrm{H}, J=14.6 \mathrm{~Hz}, \mathrm{CHHPh}), 4.54(\mathrm{dd}, 1 \mathrm{H}, J=$ $8.4,2.2 \mathrm{~Hz}, \mathrm{OCHH}), 4.67(\mathrm{~d}, 1 \mathrm{H}, J=18.4 \mathrm{~Hz}, \mathrm{CH} H), 4.88$ $(\mathrm{d}, 1 \mathrm{H}, J=14.6 \mathrm{~Hz}, \mathrm{CH} H \mathrm{Ph}), 7.14-7.26(\mathrm{~m}, 2 \mathrm{H}, \operatorname{Ar} H)$, 7.27-7.37 (m, 5H, ArH), 8.01-8.12 (m, 2H, ArH) ${ }^{13} \mathrm{C}$ NMR $\left(100 \mathrm{MHz}, \mathrm{CDCl}_{3}\right): \delta=45.9(\mathrm{t}), 48.5(\mathrm{t}), 50.5(\mathrm{t}), 51.2(\mathrm{~d})$, $68.3(\mathrm{t}), 123.8$ (d), 124.6 (d), 128.1 (d), 128.3 (d), 128.4 (d), 128.9 (d), 135.7 (s), 142.7 (s), 151.8 (s), 155.3 (s), 164.5 (s); MS: $m / z=366\left(\mathrm{M}^{+}\right)$; anal. calcd. for $\mathrm{C}_{19} \mathrm{H}_{18} \mathrm{~N}_{4} \mathrm{O}_{4}: \mathrm{C} 62.29, \mathrm{H}$ 4.95, N 15.29; found: C 62.51, H 5.22, N 15.37. 
7-Benzyl-3-(2-nitrophenylimino)-1,7,8,8a-tetrahydro-3Hoxazolo[3,4-a]pyrazine-6(5H)-one (2i): Yield: $74 \%$; pale yellow solid; $\mathrm{mp} 132^{\circ} \mathrm{C}$. IR: $v=1757,1585,1511,1356 \mathrm{~cm}^{-1}$. ${ }^{1} \mathrm{H}$ NMR $\left(400 \mathrm{MHz}, \mathrm{CDCl}_{3}\right): \delta=3.26(\mathrm{dd}, 1 \mathrm{H}, \quad J=11.5$, $4.0 \mathrm{~Hz}, \mathrm{NCHH}), 3.48$ (dd, $1 \mathrm{H}, J=11.5,9.4 \mathrm{~Hz}, \mathrm{NCH} H), 3.98$ $(\mathrm{dd}, 1 \mathrm{H}, J=8.7,3.8 \mathrm{~Hz}, \mathrm{OCHH}), 4.06(\mathrm{~d}, 1 \mathrm{H}, J=18.4 \mathrm{~Hz}$, $\mathrm{CH}), 4.01-4.08(\mathrm{~m}, 1 \mathrm{H}, \mathrm{NCH}), 4.39(\mathrm{dd}, 1 \mathrm{H}, J=8.7$, $7.1 \mathrm{~Hz}, \mathrm{OCH} H), 4.43(\mathrm{~d}, 1 \mathrm{H}, J=14.1 \mathrm{~Hz}, \mathrm{CH} H \mathrm{Ph}), 4.66$ (d, $1 \mathrm{H}, J=18.3 \mathrm{~Hz}, \mathrm{CH} H), 4.96(\mathrm{~d}, 1 \mathrm{H}, J=14.5 \mathrm{~Hz}, \mathrm{C} H \mathrm{HPh})$, 7.07-7.12 (m, 2H, ArH), 7.27-7.45 (m, 6H, ArH), $7.87(\mathrm{~d}$, $1 \mathrm{H}, J=7.5 \mathrm{~Hz}, \mathrm{Ar} H) ;{ }^{13} \mathrm{C} \mathrm{NMR}\left(100 \mathrm{MHz}, \mathrm{CDCl}_{3}\right): \delta=46.0$ $(\mathrm{t}), 48.5(\mathrm{t}), 50.4(\mathrm{t}), 51.9(\mathrm{~d}), 67.7(\mathrm{t}), 122.5(\mathrm{~d}), 124.9(\mathrm{~d})$, 126.6 (d), 128.0 (d), 128.2 (d), 128.9 (d), 133.3 (d), 135.7 (s), 142.3 (s), 143.6 (s), 152.3 (s), 164.6 (s); MS: $m / z=366\left(\mathrm{M}^{+}\right)$; anal. calcd. for $\mathrm{C}_{19} \mathrm{H}_{18} \mathrm{~N}_{4} \mathrm{O}_{4}$ : C 62.29, $\mathrm{H}$ 4.95, N 15.29; found: C 62.16, H 5.09, N 15.51.

7-Benzyl-3-(4-methoxyphenylimino)-1,7,8,8a-tetrahydro3H-oxazolo[3,4-a]pyrazine-6(5H)-one (2j): Yield: 77\%; white solid; $\mathrm{mp} 136^{\circ} \mathrm{C}$. IR: $v=1761,1586,1253 \mathrm{~cm}^{-1}$; ${ }^{1} \mathrm{H} \mathrm{NMR}\left(400 \mathrm{MHz}, \mathrm{CDCl}_{3}\right): \delta=3.26(\mathrm{dd}, 1 \mathrm{H}, \quad J=11.7$, $3.6 \mathrm{~Hz}, \mathrm{NCHH}), 3.36(\mathrm{dd}, 1 \mathrm{H}, J=11.1,10.4 \mathrm{~Hz}, \mathrm{NCH} H)$, 3.75-3.80 (m, 4H, OCHH, $\left.\mathrm{OCH}_{3}\right), 3.95-4.03(\mathrm{~m}, 2 \mathrm{H}, \mathrm{CHH}$, $\mathrm{NCH}), 4.26-4.46(\mathrm{~m}, 1 \mathrm{H}, \mathrm{OCH} H), 4.47(\mathrm{~d}, 1 \mathrm{H}, J=14.7 \mathrm{~Hz}$, $\mathrm{CHHPh}), 4.67(\mathrm{~d}, 1 \mathrm{H}, J=18.4 \mathrm{~Hz}, \mathrm{CH} H), 4.87$ (d, $1 \mathrm{H}, J=$ $14.6 \mathrm{~Hz}, \mathrm{CH} H \mathrm{Ph}), 6.80(\mathrm{~d}, 2 \mathrm{H}, J=8.8 \mathrm{~Hz}, \operatorname{Ar} H), 7.03$ (d, $2 \mathrm{H}, J=8.7 \mathrm{~Hz}, \operatorname{Ar} H)$ 7.27-7.36 (m, 6H, ArH); ${ }^{13} \mathrm{C} \mathrm{NMR}$ $\left(100 \mathrm{MHz}, \mathrm{CDCl}_{3}\right): \delta=46.2(\mathrm{t}), 48.4(\mathrm{t}), 50.3(\mathrm{t}), 51.2(\mathrm{~d})$, 55.4 (q), 67.7 (t), 113.9 (d), 122.7 (d), 127.9 (d), 128.5 (d), 129.4 (d), 135.6 (s), 139.5 (s), 150.5 (s), 155.3 (s), 165.2 (s); MS: $m / z=351\left(\mathrm{M}^{+}\right)$; anal. calcd. for $\mathrm{C}_{20} \mathrm{H}_{21} \mathrm{~N}_{3} \mathrm{O}_{3}: \mathrm{C}$ 68.36, $\mathrm{H}$ 6.02, N 11.96; found: C 68.07, H 6.12, N 12.11.

\section{General Procedure for the Diamination Reactions}

A mixture of $\mathrm{PdCl}_{2}(\mathrm{MeCN})_{2}(0.1 \mathrm{mmol}), \mathrm{CuCl}_{2}(3 \mathrm{mmol})$, $\mathrm{K}_{2} \mathrm{CO}_{3}(3 \mathrm{mmol})$, and urea $\mathbf{1}(1 \mathrm{mmol})$ in DMF $(10 \mathrm{~mL})$ was heated at $150^{\circ} \mathrm{C}$ for $15 \mathrm{~min}$ under microwave irradiation. Brine $(15 \mathrm{~mL})$ was added and the mixture was extracted with $\mathrm{CH}_{2} \mathrm{Cl}_{2}(3 \times 15 \mathrm{~mL})$. The organic phase was dried over $\mathrm{Na}_{2} \mathrm{SO}_{4}$ and the solvent removed under reduced pressure. The crude mixture was purified by silica gel column chromatography (petroleum ether/AcOEt 3:7).

7-Cyclohexyl-2-(phenyl)-1,7,8,8a-tetrahydroimidazo[1,5a]pyrazine-3,6(2H,5H)-dione (3a): Yield: $78 \%$; beige solid; $\mathrm{mp} 147^{\circ} \mathrm{C}$. IR: $v=1753,1722 \mathrm{~cm}^{-1}$; ${ }^{1} \mathrm{H} \mathrm{NMR}(400 \mathrm{MHz}$ $\left.\mathrm{CDCl}_{3}\right): \delta=1.25-1.82\left[\mathrm{~m}, 10 \mathrm{H},\left(\mathrm{CH}_{2}\right)_{5}\right], 3.23(\mathrm{dd}, 1 \mathrm{H}, J=$

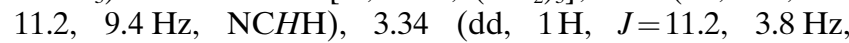
$\mathrm{NCH} H), 3.55(\mathrm{dd}, 1 \mathrm{H}, J=9.1,2.6 \mathrm{~Hz}, \mathrm{NCHH}), 3.82(\mathrm{~d}, J=$ $18.3 \mathrm{~Hz}, 1 \mathrm{H}, \mathrm{CHH}), 3.91-3.98(\mathrm{~m}, 1 \mathrm{H}, \mathrm{NCH}), 4.01(\mathrm{dd}, 1 \mathrm{H}$, $J=9.1,7.6 \mathrm{~Hz}, \mathrm{NCH} H), 4.49(\mathrm{~d}, 1 \mathrm{H}, J=18.4 \mathrm{~Hz}, \mathrm{CHH})$, 4.50-4.56 [m, 1 H, NCH $\left.\left(\mathrm{CH}_{2}\right)_{5}\right], 7.04-7.09(\mathrm{~m}, 1 \mathrm{H}, \mathrm{ArH})$, 7.27-7.35 (m, 2H, ArH), $7.53(\mathrm{~d}, 2 \mathrm{H}, J=8.8 \mathrm{~Hz}, \operatorname{Ar} H)$; ${ }^{13} \mathrm{C} \mathrm{NMR}\left(100 \mathrm{MHz}, \mathrm{CDCl}_{3}\right): \delta=25.3(\mathrm{t}), 25.4(\mathrm{t}), 25.5(\mathrm{t})$, $29.3(\mathrm{t}), 29.6(\mathrm{t}), 44.3(\mathrm{t}), 44.7(\mathrm{t}), 46.2(\mathrm{t}), 47.3(\mathrm{~d}), 52.4(\mathrm{~d})$, 117.43 (d), 123.1 (d), 128.9 (d), 139.7 (s), 155.8 (s), 164.5 (s); MS: $m / z=313\left(\mathrm{M}^{+}\right)$; anal. calcd. for $\mathrm{C}_{18} \mathrm{H}_{23} \mathrm{~N}_{3} \mathrm{O}_{2}: \mathrm{C} 68.98, \mathrm{H}$ 7.40, N 13.41; found: C 69.27, H 7.17, N 13.60.

7-Cyclohexyl-2-(4-chlorophenyl)-1,7,8,8a-tetrahydroimidazo[1,5-a]pyrazine-3,6(2H,5H)-dione (3b): Yield: $73 \%$; beige solid; $\mathrm{mp} 161^{\circ} \mathrm{C}$. IR: $v=1750,1724,1085 \mathrm{~cm}^{-1}$; ${ }^{1} \mathrm{H} N M R \quad\left(400 \mathrm{MHz}, \quad \mathrm{CDCl}_{3}\right): \delta=1.26-1.83 \quad[\mathrm{~m}, \quad 10 \mathrm{H}$, $\left.\left(\mathrm{CH}_{2}\right)_{5}\right], 3.19-3.25(\mathrm{~m}, 1 \mathrm{H}, \mathrm{NCHH}), 3.37(\mathrm{~d}, 1 \mathrm{H}, J=9.4$,
$3.2 \mathrm{~Hz}, \mathrm{NCH} H), 3.54$ (dd, $1 \mathrm{H}, J=9.4,2.6 \mathrm{~Hz}, \mathrm{NCHH}), 3.85$ $(\mathrm{d}, 1 \mathrm{H}, J=18.6 \mathrm{~Hz}, \mathrm{CHH}), 3.97-4.03(\mathrm{~m}, 2 \mathrm{H}, \mathrm{NCHH}$, $\mathrm{NCH}), 4.50(\mathrm{~d}, 1 \mathrm{H}, J=18.6 \mathrm{~Hz}, \mathrm{CHH}), 4.50-4.56[\mathrm{~m}, 1 \mathrm{H}$, $\left.\mathrm{NCH}\left(\mathrm{CH}_{2}\right)_{5}\right], 7.24(\mathrm{~d}, 2 \mathrm{H}, J=8.4 \mathrm{~Hz}, \mathrm{Ar} H), 7.34$ (d, $2 \mathrm{H}, J=$ $8.6 \mathrm{~Hz}, \mathrm{Ar} H) ;{ }^{13} \mathrm{C}$ NMR $\left(100 \mathrm{MHz}, \mathrm{CDCl}_{3}\right): \delta=25.3(\mathrm{t}), 25.4$ $(\mathrm{t}), 25.5(\mathrm{t}), 29.3(\mathrm{t}), 29.7(\mathrm{t}), 44.5(\mathrm{t}), 44.7(\mathrm{t}), 46.1(\mathrm{t}), 47.7$ (d), 52.5 (d), 121.1 (d), 127.9 (s), 128.9 (d), 137.7 (s), 155.5 (s), 164.3 (s); MS: $m / z=347\left(\mathrm{M}^{+}\right)$; anal. calcd. for $\mathrm{C}_{18} \mathrm{H}_{22} \mathrm{ClN}_{3} \mathrm{O}_{2}$ : C 62.15, H 6.37, N 12.08; found: C 61.99, $\mathrm{H}$ 6.61, N 12.29.

7-Cyclohexyl-2-(4-nitrophenyl)-1,7,8,8a-tetrahydroimidazo[1,5-a]pyrazine-3,6(2H,5H)-dione (3c): Yield: $78 \%$; brown solid; $\mathrm{mp} 162^{\circ} \mathrm{C}$. IR: $v=1757,1726,1536,1361 \mathrm{~cm}^{-1}$; ${ }^{1} \mathrm{H}$ NMR $\quad\left(400 \mathrm{MHz}, \quad \mathrm{CDCl}_{3}\right): \delta=1.12-1.81 \quad[\mathrm{~m}, \quad 10 \mathrm{H}$, $\left.\left(\mathrm{CH}_{2}\right)_{5}\right], 3.26(\mathrm{dd}, 1 \mathrm{H}, J=11.5,9.6 \mathrm{~Hz}, \mathrm{NCHH}), 3.44(\mathrm{dd}$, $1 \mathrm{H}, J=11.5,3.1 \mathrm{~Hz}, \mathrm{NCH} H), 3.64(\mathrm{dd}, 1 \mathrm{H}, J=9.2,2.6 \mathrm{~Hz}$, $\mathrm{NCHH}), 3.87(\mathrm{~d}, 1 \mathrm{H}, J=18.3 \mathrm{~Hz}, \mathrm{CHH}), 4.04-4.10(\mathrm{~m}, 2 \mathrm{H}$, $\mathrm{NCHH}, \mathrm{NCH}), 4.50(\mathrm{~d}, 1 \mathrm{H}, J=18.3 \mathrm{~Hz}, \mathrm{CHH}), 4.48-4.54$ $\left[\mathrm{m}, 1 \mathrm{H}, \mathrm{NCH}\left(\mathrm{CH}_{2}\right)_{5}\right], 7.71(\mathrm{~d}, 2 \mathrm{H}, J=9.0 \mathrm{~Hz}, \mathrm{ArH}), 7.20(\mathrm{~d}$, $2 \mathrm{H}, J=9.0 \mathrm{~Hz}, \operatorname{Ar} H) ;{ }^{13} \mathrm{C} \mathrm{NMR}\left(100 \mathrm{MHz}, \mathrm{CDCl}_{3}\right): \delta=25.3$ $(\mathrm{t}), 25.4(\mathrm{t}), 25.5(\mathrm{t}), 29.3(\mathrm{t}), 29.7(\mathrm{t}), 44.6(\mathrm{t}), 44.8(\mathrm{t}), 46.0$ (t), 47.5 (d), 52.6 (d), 116.2 (d),125.0 (d), $142.3(\mathrm{~s}), 145.4(\mathrm{~s})$, 154.8 (s), 163.8 (s); MS: $m / z=358\left(\mathrm{M}^{+}\right)$; anal. calcd. for $\mathrm{C}_{18} \mathrm{H}_{22} \mathrm{~N}_{4} \mathrm{O}_{4}$ : C 60.32, H 6.19, N 15.63; found: $\mathrm{C} 60.06, \mathrm{H}$ 6.37, N 15.70.

7-Cyclohexyl-2-(2-nitrophenyl)-1,7,8,8a-tetrahydroimidazo[1,5-a]pyrazine-3,6(2H,5H)-dione (3d): Yield: $80 \%$; white solid; $\mathrm{mp} 141^{\circ} \mathrm{C}$. IR: $v=1751,1729,1513,1356 \mathrm{~cm}^{-1}$; ${ }^{1} \mathrm{H} \mathrm{NMR} \quad\left(400 \mathrm{MHz}, \quad \mathrm{CDCl}_{3}\right): \quad \delta=1.11-1.80 \quad[\mathrm{~m}, \quad 10 \mathrm{H}$, $\left.\left(\mathrm{CH}_{2}\right)_{5}\right], 3.37$ (dd, $\left.1 \mathrm{H}, J=12.1,3.8 \mathrm{~Hz}, \mathrm{NCHH}\right), 3.46-3.49$ $(\mathrm{m}, 1 \mathrm{H}, \mathrm{NCH} H), 3.63(\mathrm{dd}, 1 \mathrm{H}, J=9.1,2.3 \mathrm{~Hz}, \mathrm{NCHH}), 3.64$ $(\mathrm{dd}, 1 \mathrm{H}, J=9.1,2.6 \mathrm{~Hz}, \mathrm{NCHH}), 3.83(\mathrm{~d}, 1 \mathrm{H}, J=18.3 \mathrm{~Hz}$, $\mathrm{CHH}), 3.95-3.05(\mathrm{~m}, 1 \mathrm{H}, \mathrm{NCH}), 4.07-4.11(\mathrm{~m}, 1 \mathrm{H}, \mathrm{NCH} H)$, $4.43(\mathrm{~d}, 1 \mathrm{H}, J=18.4 \mathrm{~Hz}, \mathrm{CH} H), 4.48-4.59[\mathrm{~m}, 1 \mathrm{H}, \mathrm{NCH}-$ $\left(\mathrm{CH}_{2}\right)_{5}$ ], 7.34-7.39 (m, 2H, $\left.\mathrm{ArH}\right), 7.61-7.66(\mathrm{~m}, 1 \mathrm{H}, \mathrm{ArH})$, $7.93(\mathrm{dd}, 1 \mathrm{H}, J=8.1,1.4 \mathrm{~Hz}, \mathrm{Ar} H) ;{ }^{13} \mathrm{C} \mathrm{NMR}(100 \mathrm{MHz}$, $\left.\mathrm{CDCl}_{3}\right): \delta=25.3(\mathrm{t}), 25.4(\mathrm{t}), 25.6(\mathrm{t}), 29.3(\mathrm{t}), 29.7(\mathrm{t}), 43.9$ $(\mathrm{t}), 44.9(\mathrm{t}), 48.2(\mathrm{t}), 48.9$ (d), $52.6(\mathrm{~d}), 125.7(\mathrm{~d}), 126.9$ (d), 127.0 (d), 132.4 (s), 133.7 (d), 145.6 (s), 156.1 (s), 164.2 (s); MS: $m / z=358\left(\mathrm{M}^{+}\right)$; anal. calcd. for $\mathrm{C}_{18} \mathrm{H}_{22} \mathrm{~N}_{4} \mathrm{O}_{4}: \mathrm{C} 60.32, \mathrm{H}$ 6.19, N 15.63; found: C 60.45, H 5.90, N 15.44.

7-Cyclohexyl-2-(4-methoxyphenyl)-1,7,8,8a-tetrahydroimidazo[1,5-a]pyrazine-3,6(2H,5H)-dione (3e): Yield: 79\%; pale yellow solid; $\mathrm{mp} 196^{\circ} \mathrm{C}$. IR: $v=1755,1728,1253 \mathrm{~cm}^{-1}$; ${ }^{1} \mathrm{H}$ NMR $\quad\left(400 \mathrm{MHz}, \quad \mathrm{CDCl}_{3}\right): \quad \delta=1.09-1.80 \quad[\mathrm{~m}, \quad 10 \mathrm{H}$, $\left(\mathrm{CH}_{2}\right)_{5}$ ], 3.24-3.30 (m, 1 H, NCHH), $3.33(\mathrm{dd}, 1 \mathrm{H}, J=10.9$, $4.0 \mathrm{~Hz}, \mathrm{NCH} H), 3.52(\mathrm{dd}, 1 \mathrm{H}, J=10.9,2.5 \mathrm{~Hz}, \mathrm{NCHH}), 3.80$ $\left(\mathrm{s}, 3 \mathrm{H}, \mathrm{OCH}_{3}\right), 3.83(\mathrm{~d}, 1 \mathrm{H}, J=18.5 \mathrm{~Hz}, \mathrm{CHH}), 3.90-4.02$ $(\mathrm{m}, 2 \mathrm{H}, \mathrm{NCHH}, \mathrm{NCH}), 4.50(\mathrm{~d}, 1 \mathrm{H}, J=18.5 \mathrm{~Hz}, \mathrm{CHH})$, 4.51-4.56 [m, 1H, NCH( $\left.\left(\mathrm{CH}_{2}\right)_{5}\right], 6.89(\mathrm{~d}, 2 \mathrm{H}, J=9.1 \mathrm{~Hz}$, $\operatorname{Ar} H), 7.43(\mathrm{~d}, 2 \mathrm{H}, J=9.1 \mathrm{~Hz}, \operatorname{Ar} H) ;{ }^{13} \mathrm{C}$ NMR $(100 \mathrm{MHz}$, $\left.\mathrm{CDCl}_{3}\right): \delta=25.3(\mathrm{t}), 25.4(\mathrm{t}), 25.5(\mathrm{t}), 29.3(\mathrm{t}), 29.7(\mathrm{t}), 44.2$ $(\mathrm{t}), 44.8(\mathrm{t}), 46.7(\mathrm{t}), 47.8(\mathrm{~d}), 52.4(\mathrm{~d}), 55.5(\mathrm{q}), 114.2$ (d),119.6 (d), 133.0 (s), 155.7 (s), 156.2 (s), 164.6 (s); MS: $m$ / $z=343\left(\mathrm{M}^{+}\right)$; anal. calcd. for $\mathrm{C}_{19} \mathrm{H}_{25} \mathrm{~N}_{3} \mathrm{O}_{3}$ : C 66.45, $\mathrm{H}$ 7.34, N 12.24; found: C 66.46, H 7.04, N 12.02 .

7-Benzyl-2-(phenyl)-1,7,8,8a-tetrahydroimidazo[1,5-a]pyrazine-3,6(2H,5H)-dione (3f): Yield: $74 \%$; white solid; mp $158^{\circ} \mathrm{C}$. IR: $v=1759, \quad 1720 \mathrm{~cm}^{-1} ;{ }^{1} \mathrm{H}$ NMR $\quad(400 \mathrm{MHz}$, $\left.\mathrm{CDCl}_{3}\right): \delta=3.25(\mathrm{dd}, 1 \mathrm{H}, J=11.8,3.8 \mathrm{~Hz}, \mathrm{NCHH}), 3.35-$ $3.45(\mathrm{~m}, 1 \mathrm{H}, \mathrm{NCH} H), 3.47(\mathrm{dd}, 1 \mathrm{H}, J=8.6,2.4 \mathrm{~Hz}, \mathrm{NCHH})$, $3.93(\mathrm{~d}, J=18.5 \mathrm{~Hz}, 1 \mathrm{H}, \mathrm{CHH}), 3.96-4.03(\mathrm{~m}, 2 \mathrm{H}, \mathrm{NCH}$, 
$\mathrm{NCH}), 4.49$ (d, $J=14.6 \mathrm{~Hz}, 1 \mathrm{H}, \mathrm{CHHPh}), 4.62(\mathrm{~d}, 1 \mathrm{H}, J=$ $18.5 \mathrm{~Hz}, \mathrm{CH} H), 4.82(\mathrm{~d}, 1 \mathrm{H}, J=14.6 \mathrm{~Hz}, \mathrm{CH} H \mathrm{Ph}), 7.07-7.09$ $(\mathrm{m}, 1 \mathrm{H}, \operatorname{Ar} H), 7.26-7.36(\mathrm{~m}, 7 \mathrm{H}, \operatorname{Ar} H), 7.51(\mathrm{dd}, 2 \mathrm{H}, J=$ 8.8, $1.1 \mathrm{~Hz}, \mathrm{ArH}) ;{ }^{13} \mathrm{C} \mathrm{NMR}\left(100 \mathrm{MHz}, \mathrm{CDCl}_{3}\right): \delta=44.5(\mathrm{t})$, $46.0(\mathrm{t}), 47.3$ (d), $49.3(\mathrm{t}), 50.4(\mathrm{t}), 117.5$ (d), 123.1 (d), 123.1 (d), 128.0 (d), 128.2 (d), 128.5 (d), 128.6 (d), 128.9 (d), 129.0 (d), 135.8 (s), 139.6 (s), 155.8 (s), 165.3 (s); MS: $m / z=321$ $\left(\mathrm{M}^{+}\right)$; anal. calcd. for $\mathrm{C}_{19} \mathrm{H}_{19} \mathrm{~N}_{3} \mathrm{O}_{2}$ : C $71.01 \mathrm{~m}, \mathrm{H} 5.96, \mathrm{~N}$ 13.08; found: C 70.88, H 6.19, N, 12.76 .

7-Benzyl-2-(4-chlorophenyl)-1,7,8,8a-tetrahydroimidazo[1,5-a]pyrazine-3,6(2H,5H)-dione (3g): Yield: 72\%; pale yellow solid; $\mathrm{mp} 167^{\circ} \mathrm{C}$. IR: $v=1757,1725,1102 \mathrm{~cm}^{-1}$; ${ }^{1} \mathrm{H}$ NMR $\left(400 \mathrm{MHz}, \mathrm{CDCl}_{3}\right): \delta=3.27(\mathrm{dd}, 1 \mathrm{H}, \quad J=11.9$, $4.1 \mathrm{~Hz}, \mathrm{NCHH}), 3.34-3.40(\mathrm{~m}, 1 \mathrm{H}, \mathrm{NCH} H), 3.44(\mathrm{dd}, 1 \mathrm{H}$, $J=8.9,2.8 \mathrm{~Hz}, \mathrm{NCHH}), 3.93(\mathrm{~d}, J=18.5 \mathrm{~Hz}, 1 \mathrm{H}, \mathrm{CHH})$, 3.95-3.99 (m, 2H, NCHH, NCH), $4.49(\mathrm{~d}, J=14.6 \mathrm{~Hz}, 1 \mathrm{H}$, $\mathrm{CHHPh}), 4.62(\mathrm{~d}, 1 \mathrm{H}, J=18.5 \mathrm{~Hz}, \mathrm{CH} H), 4.82(\mathrm{~d}, 1 \mathrm{H}, J=$ $14.6 \mathrm{~Hz}, \mathrm{CH} H \mathrm{Ph}), 7.25-7.36(\mathrm{~m}, 7 \mathrm{H}, \mathrm{ArH}), 7.46(\mathrm{dd}, 2 \mathrm{H}$, $J=7.0,2.0 \mathrm{~Hz}, \operatorname{Ar} H) ;{ }^{13} \mathrm{C} \mathrm{NMR}\left(100 \mathrm{MHz}, \mathrm{CDCl}_{3}\right): \delta=44.5$ $(\mathrm{t}), 46.0(\mathrm{t}), 47.3(\mathrm{~d}), 49.3(\mathrm{t}), 50.4(\mathrm{t}), 118.5(\mathrm{~d}), 128.0(\mathrm{~d})$, 128.3 (d), 128.8 (d), 128.9 (d), 133.8 (s), 135.8 (s), 138.2 (s), 155.5 (s), 165.1 (s); MS: $m / z=335\left(\mathrm{M}^{+}\right)$; anal. calcd. for $\mathrm{C}_{19} \mathrm{H}_{18} \mathrm{ClN}_{3} \mathrm{O}_{2}$ : C 64.13, H 5.10, N 11.81; found: $\mathrm{C} 64.08, \mathrm{H}$ 4.82, N 12.09 .

7-Benzyl-2-(4-nitrophenyl)-1,7,8,8a-tetrahydroimidazo[1,5-a]pyrazine-3,6(2H,5H)-dione (3h): Yield: $76 \%$; pale yellow solid; mp $186^{\circ} \mathrm{C}$. IR: $v=1758,1729.1524,1348 \mathrm{~cm}^{-1}$; ${ }^{1} \mathrm{H}$ NMR (400 MHz, $\left.\mathrm{CDCl}_{3}\right): \delta=3.27-3.28(\mathrm{~m}, 2 \mathrm{H}, \mathrm{NCH} H)$, $3.56(\mathrm{dd}, 1 \mathrm{H}, J=11.8,3.8 \mathrm{~Hz}, \mathrm{NCHH}), 3.96(\mathrm{~d}, J=18.5 \mathrm{~Hz}$, $1 \mathrm{H}, \mathrm{CHH}), 4.02-4.10(\mathrm{~m}, 2 \mathrm{H}, \mathrm{NCH} H, \mathrm{NCH}), 4.53(\mathrm{~d}, J=$ $14.5 \mathrm{~Hz}, 1 \mathrm{H}, \mathrm{CHHPh}), 4.62(\mathrm{~d}, 1 \mathrm{H}, J=18.5 \mathrm{~Hz}, \mathrm{CH} H), 4.81$ (d, $1 \mathrm{H}, J=14.5 \mathrm{~Hz}, \mathrm{CH} H \mathrm{Ph}), 7.27-7.37$ (m, 5 H, ArH), 7.68 (d, $2 \mathrm{H}, J=9.3 \mathrm{~Hz}, \operatorname{Ar} H), 8.21(\mathrm{~d}, 2 \mathrm{H}, J=9.4 \mathrm{~Hz}, \operatorname{Ar} H)$; ${ }^{13} \mathrm{C}$ NMR $\left(100 \mathrm{MHz} \mathrm{CDCl}_{3}\right): \delta=44.4(\mathrm{t}), 45.8(\mathrm{t}), 47.0(\mathrm{~d})$, $49.6(\mathrm{t}), 50.5$ (t), 116.3 (d), 125.0 (d), 128.1 (d), 128.3 (d), 129.1 (d), 135.6 (s), 145.2 (s), 152.7 (s), 154.7 (s), 164.6 (s); MS: $m / z=366\left(\mathrm{M}^{+}\right)$; anal. calcd. for $\mathrm{C}_{19} \mathrm{H}_{18} \mathrm{~N}_{4} \mathrm{O}_{4}: \mathrm{C} 62.29, \mathrm{H}$ 4.95, N 15.29; found: C 62.44, H 4.72, N 15.42.

7-Benzyl-2-(2-nitrophenyl)-1,7,8,8a-tetrahydroimidazo[1,5-a]pyrazine-3,6(2H,5H)-dione (3i): Yield: 78\%; white solid; $\mathrm{mp} 163^{\circ} \mathrm{C}$. IR: $v=1754,1721,1514,1355 \mathrm{~cm}^{-1}$; ${ }^{1} \mathrm{H} \mathrm{NMR}\left(400 \mathrm{MHz}, \mathrm{CDCl}_{3}\right): \delta=3.26(\mathrm{dd}, 1 \mathrm{H}, \quad J=9.3$, $3.6 \mathrm{~Hz}, \mathrm{NCHH}), 3.53-3.63(\mathrm{~m}, 2 \mathrm{H}, \mathrm{NCHH}, \mathrm{NCH} H), 3.93$ $(\mathrm{d}, J=18.5 \mathrm{~Hz}, 1 \mathrm{H}, \mathrm{CHH}), 4.00-4.10(\mathrm{~m}, 2 \mathrm{H}, \mathrm{NCH} H$, $\mathrm{NCH}), 4.41(\mathrm{~d}, J=14.7 \mathrm{~Hz}, 1 \mathrm{H}, \mathrm{CHHPh}), 4.56(\mathrm{~d}, 1 \mathrm{H}, J=$ $18.5 \mathrm{~Hz}, \mathrm{CH} H), 4.96(\mathrm{~d}, 1 \mathrm{H}, J=14.7 \mathrm{~Hz}, \mathrm{CH} H \mathrm{Ph}), 7.28-7.38$ $(\mathrm{m}, 7 \mathrm{H}, \operatorname{Ar} H), 7.61-7.66(\mathrm{~m}, 1 \mathrm{H}, \operatorname{Ar} H), 7.93(\mathrm{dd}, 1 \mathrm{H}, J=$ $8.1,1.4 \mathrm{~Hz}, \mathrm{Ar} H) ;{ }^{13} \mathrm{C}$ NMR $\left(100 \mathrm{MHz}, \mathrm{CDCl}_{3}\right): \delta=44.7(\mathrm{t})$ $48.0(\mathrm{t}), 48.5(\mathrm{~d}), 48.8(\mathrm{t}), 50.3(\mathrm{t}), 122.5(\mathrm{~d}), 126.9(\mathrm{~d}), 127.0$ (d), 127.9 (d), 128.2 (d), 128.9 (d), 132.4 (s), 133.3 (d), 133.6 (d), 135.9 (s), 145.6 (s), 156.0 (s), 164.9 (s); MS: $m / z=366$ $\left(\mathrm{M}^{+}\right)$; anal. calcd. for $\mathrm{C}_{19} \mathrm{H}_{18} \mathrm{~N}_{4} \mathrm{O}_{4}$ : C 62.29, $\mathrm{H}$ 4.95, N 15.29; found: $\mathrm{C}$ 62.07, $\mathrm{H}$ 5.12, N 15.22.

7-Benzyl-2-(4-methoxyphenyl)-1,7,8,8a-tetrahydroimidazo[1,5-a]pyrazine-3,6(2H,5H)-dione $(3 \mathbf{j})$ : Yield: $73 \%$; beige solid; mp $225^{\circ} \mathrm{C}$. IR: $v=1755,1724,1261 \mathrm{~cm}^{-1} ;{ }^{1} \mathrm{H} \mathrm{NMR}$ $\left(400 \mathrm{MHz}, \quad \mathrm{CDCl}_{3}\right): \delta=3.24(\mathrm{dd}, \quad 1 \mathrm{H}, \quad J=11.8, \quad 4.0 \mathrm{~Hz}$, $\mathrm{NCHH}), 3.37-3.44(\mathrm{~m}, 2 \mathrm{H}, \mathrm{NCH} H, \mathrm{NCHH}), 3.79(\mathrm{~s}, 3 \mathrm{H}$, $\left.\mathrm{OCH}_{3}\right), 3.91(\mathrm{~d}, J=18.5 \mathrm{~Hz}, 1 \mathrm{H}, \mathrm{CHH}), 3.92-3.98(\mathrm{~m}, 2 \mathrm{H}$, $\mathrm{NCH} H, \mathrm{NCH}), 4.50(\mathrm{~d}, J=14.6 \mathrm{~Hz}, 1 \mathrm{H}, \mathrm{CHHPh}), 4.61(\mathrm{~d}$, $1 \mathrm{H}, J=18.5 \mathrm{~Hz}, \mathrm{CH} H), 4.82(\mathrm{~d}, 1 \mathrm{H}, J=14.6 \mathrm{~Hz}, \mathrm{CH} H \mathrm{Ph})$, $6.88(\mathrm{~d}, 1 \mathrm{H}, J=9.1 \mathrm{~Hz}, \operatorname{Ar} H), 7.28-7.41(\mathrm{~m}, 7 \mathrm{H}, \operatorname{Ar} H)$;
${ }^{13} \mathrm{C} \mathrm{NMR}\left(100 \mathrm{MHz}, \mathrm{CDCl}_{3}\right): \delta=44.6(\mathrm{t}), 46.6(\mathrm{t}), 47.5(\mathrm{~d})$, 49.2 (t), 50.4 (t), 55.5 (q), 114.2 (d), 119.6 (d), 127.9 (d), 128.3 (d), 129.9 (d), 132.9 (s), 138.7 (s), 155.8 (s), 147.1 (s), 165.4(s); MS: $m / z=351\left(\mathrm{M}^{+}\right)$; anal. calcd. for $\mathrm{C}_{20} \mathrm{H}_{21} \mathrm{~N}_{3} \mathrm{O}_{3}: \mathrm{C}$ 68.36, H 6.02, N 11.96; found: C 68.11, H 5.76, N 12.25.

\section{Acknowledgements}

Thanks are due to the Ministero dell'Università e della Ricerca as well as to Insubria University, Milano University and Catania University for financial support.

\section{References}

[1] a) D. M. Schultz, J. P. Wolfe, Synthesis 2012, 44, 351361; b) R. I. McDonald, G. Liu, S. S. Stahl, Chem. Rev. 2011, 111, 2981-3019; c) E. M. Beccalli, G. Broggini, M. Martinelli, S. Sottocornola, Chem. Rev. 2007, 107, 5318-5365; d) S. R. Chemler, Org. Biomol. Chem. 2009, 7, 3009-3019; e) K. H. Jensen, M. S. Sigman, Org. Biomol. Chem. 2008, 6, 4083-4088; f) V. Kotov, C. C. Scarborough, S. S. Stahl, Inorg. Chem. 2007, 46, 19101923.

[2] For aminooxygenation reactions, see: a) E. J. Alexanian, C. Lee, E. J. Sorensen, J. Am. Chem. Soc. 2005, 127, 7690-7691; b) G. Liu, S. S. Stahl, J. Am. Chem. Soc. 2006, 128, 7179-7181; c) L. V. Desai, M. S. Sanford, Angew. Chem. 2007, 119, 5839-5842; Angew. Chem. Int. Ed. 2007, 46, 5737-5740; d) P. Szolcsányi, T. Gracza, Chem. Commun. 2005, 3948-3950; e) J. Alladoum, E. Vrancken, P. Mangeney, S. Roland, C. Kadouri-Puchot, Org. Lett. 2009, 11, 3746-3749; f) E. Borsini, G. Broggini, A. Fasana, S. Galli, M. Khansaa, U. Piarulli, M. Rigamonti, Adv. Synth. Catal. 2011, 353, 985-994.

[3] For diamination reactions, see: a) P. A. Sibbald, F. E. Michael, Org. Lett. 2009, 11, 1147-1149; b) G. L. J. Bar, G. C. Lloyd-Jones, K. I. Booker-Milburn, J. Am. Chem. Soc. 2005, 127, 7308-7309. For some examples to bicyclic compounds, see: c) T. Tsujihara, T. Shinohara, K. Takenaka, S. Takizawa, K. Onitsuka, M. Hatanaka, H. Sasai, J. Org. Chem. 2009, 74, 9274-9279; d) K. Muñiz, J. Am. Chem. Soc. 2007, 129, 14542-14543; e) A. Iglesias, E. Pérez, K. Muñiz, Angew. Chem. 2010, 122, 8286-8288; Angew. Chem. Int. Ed. 2010, 49, 8109-8111; f) K. Muñiz, J. Kirsch, P. Chavez, Adv. Synth. Catal. 2011, 353, 689-694; g) C. Martinez, K. Muñiz, Angew. Chem. 2012, 124, 7138-7141; Angew. Chem. Int. Ed. 2012, 51, 7031-7034; h) P. Chávez, J. Kirsch, J. Streuff, K. Muñiz, J. Org. Chem. 2012, 77, 1922-1930.

[4] For dioxygenation reactions, see: a) W. Wang, F. Wang, M. Shi, Organometallics 2010, 29, 928-933; b) A. Wang, H. Jiang, H. Chen, J. Am. Chem. Soc. 2009, 131, 38463847; c) Y. Li, D. Song, V. M. Dong, J. Am. Chem. Soc. 2008, 130, 2962-2964; d) I. Shiina, Y. Kawakita, R. Ibuka, K. Yokoyama, Y. Yamai, Chem. Commun. 2005, 4062-4064; e) M. Palík, O. Karlubíková, D. Lackovicová, A. Lásiková, T. Graczá, Tetrahedron 2010, 66, 5244-5249; f) M. Palík, O. Karlubíková, A. Lásiková, J. 
Kožíšek, T. Graczá, Eur. J. Org. Chem. 2009, 709-715; g) F. Alonso, D. Sánchez, T. Soler, M. Yus, Adv. Synth. Catal. 2008, 350, 2118-2126; h) M. J. Schultz, M. Sigman, J. Am. Chem. Soc. 2006, 128, 1460-1461.

[5] For carboamination reactions, see: a) C. F. Rosewall, P. A. Sibbald, D. V. Liskin, F. E. Michael, J. Am. Chem. Soc. 2009, 131, 9488-9489; b) C. E. Houlden, C. D. Bailey, J. Gair Ford, M. R. Gagné, G. C. Lloyd-Jones, K. I. Booker-Milburn, J. Am. Chem. Soc. 2008, 130, 10066-10067; c) C. C. Scarborough, S. S. Stahl, Org. Lett. 2006, 8, 3251-3254; d) K.-T. Yip, N.-Y. Zhu, D. Yang, Org. Lett. 2009, 11, 1911-1914; e) W. He, K.-T. Yip, N.-Y. Zhu, D. Yang, Org. Lett. 2009, 11, 56265628 ; f) B. Zhu, G.-W. Wang, Org. Lett. 2009, 11, 4334 4337; g) K.-T. Yip, M. Yang, K.-L. Law, N.-Y. Zhu, D. Yang, J. Am. Chem. Soc. 2006, 128, 3130-3131; h) Y.-Y. Liu, R.-J. Song, C.-Y. Wu, L.-B. Gong, M. Hu, Z.-Q. Wang, Y.-X. Xie, J.-H. Li, Adv. Synth. Catal. 2012, 354, 347-353.

[6] For aminohalogenation reactions, see: a) A. Lei, X. Lu, G. Liu, Tetrahedron Lett. 2004, 45, 1785-1788; b) M. R. Manzoni, T. P. Zabawa, D. Kasi, S. R. Chemler Organometallics 2004, 23, 5618-5621; c) P. Szolcsányi, T. Graczá, Tetrahedron 2006, 62, 8498-8502; d) S. D. R. Christie, A. D. Warrington, C. J. Lunniss, Synthesis 2009, 148-154; e) S. Qiu, T. Xu, J. Zhou, Y. Guo, G. Liu, J. Am. Chem. Soc. 2010, 132, 2856-2857; f) E. Borsini, G. Broggini, F. Colombo, M. Khansaa, A. Fasana, S. Galli, D. Passarella, E. Riva, S. Riva, Tetrahedron: Asymmetry 2011, 22, 264-269; g) X.-Y. Liu, P. Gao, Y.W. Shen, Y.-M. Liang, Org. Lett. 2011, 13, 4196-4199; h) G. Yin, T. Wu, G. Liu, Chem. Eur. J. 2012, 18, 451455; i) G. Broggini, V. Barbera, E. M. Beccalli, E. Borsini, S. Galli, G. Lanza, G. Zecchi, Adv. Synth. Catal. 2012, 354, 159-170.

[7] a) J. Streuff, C. H. Hövelmann, M. Nieger, K. Muñiz, J. Am. Chem. Soc. 2005, 127, 14586-14587; b) K. Muñiz, C. H. Hövelmann, J. Streuff, J. Am. Chem. Soc. 2008 , 130, 763-773.

[8] a) K. Muñiz, C. H. Hövelmann, E. Campos-Gomez, J. Barluenga, J. M. Gonzalez, J. Streuff, M. Nieger, Chem.
Asian J. 2008, 3, 776-788; b) K. Muñiz, J. Streuff, P. Chávez, C. H. Hövelmann, Chem. Asian J. 2008, 3, 1248-1255.

[9] K. Muñiz, A. Iglesias, Y. Fang, Chem. Commun. 2009, 5591-5593.

[10] H. Li, R. A. Widenhoefer, Tetrahedron 2010, 66, 48274831.

[11] a) L. Basolo, E. M. Beccalli, E. Borsini, G. Broggini, M. Khansaa, M. Rigamonti, Eur. J. Org. Chem. 2010, 1694; b) G. Broggini, E. Borsini, A. Fasana, G. Poli, F. Liron, Eur. J. Org. Chem. 2012, 3617-3624.

[12] a) T. H. Kim, G. J. Lee, J. Org. Chem. 1999, 64, 29412943; b) F. Saczewski, J. Saczewski, M. Gdaniec, Chem. Pharm. Bull. 2001, 49, 1203-1206; c) T. H. Kim, N. Lee, G. J. Lee, J. N. Kim, Tetrahedron 2001, 57, 7137-7141.

[13] Relevant crystallographic data for 2d: monoclinic, $P 2_{1} /$ $n, a=12.26(2) \AA, \quad b=9.818(7) \AA, c=15.414(9) \AA, \beta=$ $106.24(6)^{\circ}, \quad V=1781(2) \AA^{3}, \quad Z=4, \quad F(000)=760, \quad \rho=$ $1.336 \mathrm{~g} \mathrm{~cm}^{-3}, \mu(\mathrm{Mo}-\mathrm{K} \alpha)=0.096 \mathrm{~mm}^{-1}$. R and $\mathrm{wR}=$ 0.067 and 0.155 for 1914 observed reflections $;=0.119$ and 0.185 for 3213 reflections. Goodness of fit, highest peak and deepest hole $=1.038,0.4 \mathrm{e} \AA^{-3}$ and $-0.2 \mathrm{e}^{-3}$. CCDC 922353 contains the supplementary crystallographic data for this paper. These data can be obtained free of charge from The Cambridge Crystallographic Data Centre via www.ccdc.cam.ac.uk/data_request/cif.

[14] F. E. Michael, P. A. Sibbald, B. M. Cochran, Org. Lett. 2008, 10, 793-796.

[15] For the concept of transient palladium oxidation with $\mathrm{CuCl}_{2}$, see: a) H. Stangl, R. Jira, Tetrahedron Lett. 1970, 11, 3589-3592; b) O. Hamed, P. M. Henry, Organometallics 1998, 17, 5184-5189; c) C. H. Hövelmann, J. Streuff, L. Brelot, K. Muñiz, Chem. Commun. 2008, 2334-2336.

[16] For the concept of a heterobimetallic $\sigma-\mathrm{Pd} / \mathrm{Cu}$ complex, see: a) P. Szolcsányi, T. Graczá, Tetrahedron 2006, 62, 8498-8502; b) D. Zargarian, Organometallics 1991, 10, 2914-2921; c) T. Hosokawa, T. Uno, S. Inui, S.-I. Murahashi, J. Am. Chem. Soc. 1996, 118, 3990-3991. 